\title{
Locating Multiple Multiscale Acoustic Scatterers
}

\author{
Jingzhi Li* Hongyu Liu ${ }^{\dagger} \quad$ Jun Zou ${ }^{\ddagger}$
}

March 24, 2014

\begin{abstract}
We develop three inverse scattering schemes for locating multiple multiscale acoustic scatterers in a very general and practical setting. For all of the three locating schemes, only one single far-field measurement is used. The number of the multiple scatterer components may be unknown, and each scatterer component is allowed to be an inhomogeneous medium with an unknown content or an impenetrable obstacle of sound-soft, sound-hard or impedance type. Moreover, the scatterers could be multiscale, i.e., some scatterers may be of regular size, and some others may be of small size in terms of the wavelength of the detecting acoustic wave. If a scatterer component is of regular size, it is required that its shape (not necessarily its orientation, size and location) should be from an admissible class which is known in advance. The locating schemes are based on some novel indicator functions, and are computationally cheap and robust against the measurement noise. Rigorous mathematical justifications are provided for each scheme, and numerical experiments are presented to demonstrate its robustness and effectiveness.
\end{abstract}

Key Words. Inverse scattering, locating, multiscale acoustic scatterers, single far-field measurement, indicator functions

AMS subject classifications. 35P25, 35Q60, 76Q05,78A46

\section{Introduction}

In this work we are concerned with inverse scattering problems, which arise in non-invasive detecting, imaging, and remote sensing by acoustic waves. In practical applications, some unknown or inaccessible objects might be located in an otherwise homogeneous space. Each inhomogeneous object is referred to as a scatterer. One sends certain detecting wave fields, and measures the scattered/perturbed wave fields produced by the scatterer to infer some knowledge about the scatterer. The inverse scattering problem has been playing a critical role in many areas of science and technology, such as radar and sonar, non-destructive testing, remote sensing, geophysical exploration and medical imaging; see $[12,13,18,19,22,27,38,45,46]$ and the references therein. Before we proceed to discuss the new results in this work, we first present the mathematical formulation of the inverse scattering problem.

\footnotetext{
${ }^{*}$ Faculty of Science, South University of Science and Technology of China, 518055 Shenzhen, P. R. China. Email: li.jz@sustc.edu.cn

${ }^{\dagger}$ Department of Mathematics, Hong Kong Baptist University, Kowloon Tong, Hong Kong SAR. Email: hongyu.liuip@gmail.com

${ }^{\ddagger}$ Department of Mathematics, The Chinese University of Hong Kong, Shatin, N. T., Hong Kong SAR. Email zou@math. cuhk.edu.hk
} 
We shall always take the detecting wave field to be a time-harmonic plane wave of the form

$$
u^{i}(x ; d, k):=e^{i k x \cdot d}, \quad x \in \mathbb{R}^{N},
$$

where $N=2$ or 3 , and $k \in \mathbb{R}_{+}$and $d \in \mathbb{S}^{N-1}$ denote the wave number and the incident direction, respectively. Consider a bounded $C^{2}$ domain $\Omega$ in $\mathbb{R}^{N}$, which supports an inhomogeneous acoustic scatterer, and it is assumed that $\mathbb{R}^{N} \backslash \bar{\Omega}$ is connected. Depending on the acoustic materials of the scatterer, it could be either a medium or an obstacle. If $\Omega$ is an inhomogeneous medium, then it is characterized by a positive $C^{1}$ function $n(x)$ such that $\|n(x)-1\|_{L^{\infty}(\Omega)}>0$ for $x \in \bar{\Omega}$, and $n(x)$ is known as the refractive index function of the medium. The direct scattering problem corresponding to the medium $(\Omega ; n)$ is described by the Helmholtz equation:

$$
\Delta u(x)+k^{2}\left(1+\left(n^{2}(x)-1\right) \chi_{\Omega}\right) u(x)=0 \quad \text { for } x \in \mathbb{R}^{N},
$$

where $u$ is the total wave field, formed by the incident wave $u^{i}$ and the scattered wave $u^{s}$, namely

$$
u(x):=u^{i}(x)+u^{s}(x) \quad \text { for } \quad x \in \mathbb{R}^{N} .
$$

The scattered wave $u^{s}(x)$ should satisfy the physical Sommerfeld radiation condition:

$$
\lim _{|x| \rightarrow \infty}|x|^{(N-1) / 2}\left(\frac{\partial u^{s}(x)}{\partial|x|}-i k u^{s}(x)\right)=0,
$$

which holds uniformly in all directions $\hat{x}:=x /|x|, x \in \mathbb{R}^{N}$. If $\Omega$ is an obstacle, then the wave field can not penetrate and the direct scattering problem is described by

$$
\Delta u(x)+k^{2} u(x)=0 \quad \text { for } \quad x \in \mathbb{R}^{N} \backslash \bar{\Omega},
$$

where $u$ is again the total field, $u(x)=u^{i}(x)+u^{s}(x)$ for $x \in \mathbb{R}^{N} \backslash \bar{\Omega}$, and $u^{s}$ satisfies the Sommerfeld radiation condition (1.4). In addition, we should complement the obstacle scattering problem with either one of the following three boundary conditions:

$$
u=0 \quad \text { on } \partial \Omega ; \quad \frac{\partial u}{\partial \nu}=0 \quad \text { on } \partial \Omega ; \quad \frac{\partial u}{\partial \nu}+i \lambda u=0 \quad \text { on } \partial \Omega
$$

corresponding, respectively, to the case when the obstacle is sound-soft, sound-hard, and of impedance type. In (1.6), $\nu$ is the outward unit normal vector to $\partial \Omega$, and $\lambda \in C(\partial \Omega)(\lambda>0)$ stands for a surface impedance. We refer to $[36,40,41]$ for the existence of a unique $H_{l o c}^{1}$ solution to the medium scattering system (1.2)-(1.4); or the obstacle scattering system (1.3)(1.6). Particularly, $u^{s}$ admits the following asymptotic expansion as $|x| \rightarrow+\infty$ (cf. [22,41]):

$$
u^{s}(x)=\frac{e^{i k|x|}}{|x|^{\frac{N-1}{2}}} a_{k}(\hat{x}, d)+\mathcal{O}\left(\frac{1}{|x|^{\frac{N+1}{2}}}\right)
$$

where $a_{k}(\hat{x}, d)$ is often called the far-field pattern or the scattering amplitude, and $\hat{x} \in \mathbb{S}^{N-1}$ is known as the observation angle/direction, while $k$ and $d$ are the wave number and incident direction of the incident plane wave.

The inverse problems that we shall consider in this work are to recover the scatterer, namely $(\Omega, n)$ if it is a medium, or $(\Omega, \lambda)$ if it is an obstacle of impedance type, or $\Omega$ if it is a sound-soft 
or sound-hard obstacle, by the knowledge of the far-field pattern $a_{k}(\hat{x}, d)$. If one introduces an operator $\mathcal{F}$ which maps the scatterer to the corresponding far-field pattern, the inverse scattering problem can be formulated as the following operator equation

$$
\mathcal{F}(\mathcal{O})=a_{k}(\hat{x}, d), \quad k \in \mathbb{R}_{+}, \hat{x}, d \in \mathbb{S}^{N-1},
$$

where $\mathcal{O}$ represents $(\Omega, n),(\Omega, \lambda)$ or $\Omega$, depending on the type of the concerned inverse problem. It is widely known that the operator equation (1.8) is nonlinear and ill-posed (cf. [22]). In $(1.8), a_{k}(\hat{x}, d)$ is given by the measurement data which are usually recorded by some physical apparatus. The data shall be called a single measurement if $a_{k}(\hat{x}, d)$ is given for a fixed $k \in \mathbb{R}_{+}$ and a fixed $d \in \mathbb{S}^{N-1}$, but for all $\hat{x} \in \mathbb{S}^{N-1}$. That is, for a single far-field measurement, one collects the far-field data in every possible observation direction by sending a single detecting plane wave. If multiple detecting plane waves are used, e.g., many different $d$ 's or $k$ 's are used, then the corresponding scattering data shall be referred to as multiple measurements. We note that as $a_{k}(\cdot, d)$ is an analytic function on the unit sphere, it is known on the whole sphere as long as it is known on any open subset of the unit sphere by the analytic continuation.

In this work, we develop three novel numerical reconstruction schemes for the aforementioned inverse acoustic scattering problems, more specifically, for locating multiple scatterers by using the far-field data. These methods have several salient and promising features. First, all three locating schemes make use of only a single far-field measurement. As it is well-known, the inverse scattering method with a single far-field measurement is extremely challenging, with very limited theoretical and numerical advances available. We refer to $[35,37,38]$ for the related backgrounds and existing progresses in the literature. Second, our methods work in very general and practical settings. There might be multiple scatterer components of an unknown number, and each scatterer component could be an inhomogeneous medium with an unknown content or an impenetrable obstacle of sound-soft, sound-hard or impedance type. Moreover, the scatterers could be of multiple scales, which may include simultaneously both components of regular size and small size compared with the wavelength of the detecting acoustic wave. If a scatterer component is of regular size, its shape (not necessarily its orientation, size and location) is required to belong to an admissible class, which is known in advance. But the admissible class may contain many different reference scatterers. Furthermore, some reference scatterers may appear more than once in the target object, but some others might not show up. The reference scatterers might be rotated and scaled in the target object. Third, the new locating schemes are of a direct nature. They are based on some indicator functions, whose evaluations do not involve any inversions, so they are computationally very efficient and also very robust to measurement noise. For each scheme, both rigorous theoretical justifications and numerical experiments are provided.

Our study follows a similar spirit to the one of the locating methods that were recently proposed in [31] and [32] for electromagnetic (EM) scattering problems governed by the timeharmonic Maxwell equations. The methods in [31] and [32] are based on two imaging functionals, respectively, for locating small-size and regular-size EM media or perfectly conducting (PEC) obstacles. A local re-sampling technique was developed in [32] to concatenate the two imaging functionals for locating multiscale EM scatterers. In this work we shall develop three schemes, Schemes I, II and III, respectively for locating small-size, regular-size and multiscale acoustic scatterers. Due to the distinct physical nature of the acoustic scattering problems, some new ingredients and techniques are needed. In defining the imaging functional of locating small EM scatterers in [31], only the EM monopoles are involved. However, the acoustic scattering from 
small scatterers exhibit more complicated behaviors. In order to obtain the imaging functional for Scheme I that works independently of the physical properties of the underlying acoustic objects, both the acoustic monopole and dipoles should be incorporated (see definition (2.9) in next section). For locating regular-size scatterers, we present our acoustic study in a much more comprehensive manner than that in [31] and [32] for the EM case. Indeed, the regular-size EM scatterers were all assumed in [31] and [32] to be PEC obstacles, while the regular-size scatterers can be both inhomogeneous media and impenetrable obstacles of different kinds in the current acoustic case. Finally, in order to concatenate Schemes I and II to obtain Scheme III for locating multiscale acoustic scatterers, a local tuning technique is proposed. The local tuning technique generalizes the local re-sampling technique proposed in [32]. In fact, the local re-sampling technique was only used for tuning the locations of scatterers, whereas the local tuning technique here can be used for adjusting the orientations, scales as well as locations; see more discussions in Section 4. The local tuning technique concatenates Schemes I and II in a nice manner to produce Scheme III, which can be used for locating multiscale acoustic scatterers in a very general and practical setting. It is remarked that the local tuning technique can be directly extended to strengthen the method proposed in [32] for locating multiscale EM scatterers to enable it to work in a more general setting as considered here.

We would like to point out that many numerical reconstruction methods have been developed for inverse scattering problems in various scenarios, such as the linear sampling method, factorization method, MUSIC-type methods, time reversal, and topological-optimization-type methods etc.; we refer the readers to $[2,4-13,15-21,24-26,28,43-46]$ and the references therein for these methods and some other related developments. Compared with most of the existing methods, which rely on multiple scattered field measurements, the methods developed in this work are new and more general in the sense that they combine all of the following features together: only one single far-field measurement is used; the scatterers are allowed to be a multiscale mixed set of inhomogeneous media and impenetrable obstacles; rigorous mathematical justifications are established under general settings; some iterative-type refining and local tuning strategies are introduced for quantitatively improving the reconstructions. More relevant discussions on the comparisons of our method with others are provided in Sections 2 and 3.

The rest of this paper is organized as follows. In Sections 2, 3 and 4, we shall develop Schemes $I$, II and III respectively for locating multiple small-size scatterers, multiple regular-size scatterers and multiple multiscale scatterers. For each of the three schemes, rigorous mathematical justifications and numerical results are also provided.

\section{$2 \quad$ Locating small scatterers}

Throughout the rest of the paper, we assume the incident acoustic wavenumber $k=\mathcal{O}(1)$. That is, the wavelength of the incident plane wave is given by $\lambda=2 \pi / k=\mathcal{O}(1)$, hence the size of a scatterer can be expressed in terms of its Euclidean diameter. In this section, we shall develop an imaging scheme, referred to as Scheme I, to locate multiple small scatterers in terms of the incident wavelength.

\section{$2.1 \quad$ Scheme I}

We first introduce the class of small acoustic scatterers for our current study. Let $l \in \mathbb{N}$, and $D_{j}$, $1 \leq j \leq l$, be bounded simply-connected $C^{2}$ domains in $\mathbb{R}^{N}$ containing the origin. For $\rho \in \mathbb{R}_{+}$, 
we define $\rho D_{j}:=\left\{\rho x \mid x \in D_{j}\right\}$ and set

$$
\Omega_{j}^{s}=z_{j}+\rho D_{j}, \quad z_{j} \in \mathbb{R}^{N}, \quad 1 \leq j \leq l .
$$

Each $\Omega_{j}^{s}$ is referred to as a small scatterer component. If it is sound-soft or sound-hard, we further write it, respectively, as

$$
\Omega_{j}^{s, s}:=z_{j}+\rho D_{j}^{s} \quad \text { and } \quad \Omega_{j}^{s, h}:=z_{j}+\rho D_{j}^{h} .
$$

If $\Omega_{j}^{s}$ is of impedance type, we let $\lambda_{j}$ be the surface impedance on $\partial \Omega_{j}^{s}$, and denote by

$$
\left(\Omega_{j}^{s} ; \lambda_{j}\right):=\Omega_{j}^{s, i}=z_{j}+\rho D_{j}^{i},
$$

where

$$
D_{j}^{i}:=\left(D_{j} ; \lambda_{j}\left(\cdot+z_{j}\right)\right) \quad \text { and } \quad \rho D_{j}^{i}=\left(\rho D_{j} ; \lambda_{j}\left(\frac{\dot{-}}{\rho}+z_{j}\right)\right) .
$$

If $\Omega_{j}^{s}$ is an inhomogeneous medium, we let $n_{j}$ be its refractive index, and denote by

$$
\left(\Omega_{j}^{s} ; n_{j}\right):=\Omega_{j}^{s, m}=z_{j}+\rho D_{j}^{m},
$$

where

$$
D_{j}^{m}:=\left(D_{j} ; n_{j}\left(\cdot+z_{j}\right)\right) \quad \text { and } \quad \rho D_{j}^{m}:=\left(\rho D_{j} ; n_{j}\left(\frac{\dot{-}}{\rho}+z_{j}\right)\right) .
$$

In the sequel, we set

$$
\Omega^{s, t}:=\bigcup_{j=1}^{l_{t}} \Omega_{j}^{s, t}, \quad t=s, h, i \text { or } m,
$$

where $l_{t}, \rho$ and $t$ denote respectively the number of components in the scatterer $\Omega^{s, t}$, the relative size of each component in $\Omega^{s, t}$, and the type of the scatterer, which can be sound-soft, soundhard, of impedance type, or a medium. For $\Omega_{j}^{s, t}$ introduced in (2.6), we shall impose the following qualitative assumptions

$$
\rho \ll 1 \quad \text { and } \quad L=\min _{1 \leq j, j^{\prime} \leq l_{t}, j \neq j^{\prime}} \operatorname{dist}\left(z_{j}, z_{j^{\prime}}\right) \gg 1 .
$$

These conditions mean that the relative size of each scatterer component is small compared with the wavelength of the detecting/incident wave, and all the components must be well separated in the case of multiple components. It is remarked that $\rho$ and $L$ in (2.7) should be different with different type of scatterer components; see also Remark 2.1 at the end of this section. But we will always use the same $\rho$ and $L$ for the ease of notations.

In the sequel, we develop Scheme $I$ to locate the multiple components of $\Omega^{s, t}$ introduced in (2.6). The imaging scheme works in a very general and practical setting. First, we assume very little a priori knowledge of the scatterer. Both its type and the number of the components, i.e., $t$ and $l_{t}$, are not required to be known in advance. Second, if the scatterer is a medium or of impedance type, the refractive indices or the surface impedances of its components are not required to be known a priori. Third, in a certain generic situation, the underlying scatterer $\Omega^{s, t}$ could be composed of mixed-type components, namely some of its components could be media while the others are obstacles of different type. We shall give some more remarks about this point at the end of this section. Finally, we would like to point out that our numerical 
experiments could speak a bit more about the qualitative assumptions (2.7): Scheme I can produce satisfactory reconstructions, as long as the size of the scatterer is smaller than half of the detecting wavelength while the distance between any two different components is bigger than half of the detecting wavelength. Nevertheless, in the extreme situation where the distance between two scatterer components is smaller than half of the detecting wavelength, Scheme I can still produce some qualitative reconstruction of the profile of the two scatterers but it may not be able to clearly separate them; we refer to Fig. 4.4 in [31] for reconstructing two closeby electromagnetic scatterers, and Scheme I produces similar reconstructions for the current acoustic case.

We are now ready to present our first locating scheme. To begin with, we let

$$
a\left(\hat{x} ; \Omega^{s, t}\right):=a_{k}\left(\hat{x}, d ; \Omega^{s, t}\right), \quad \hat{x} \in \mathbb{S}^{N-1},
$$

denote the scattering amplitude of $\Omega^{s, t}$ in (2.6) due to a single incident plane wave $e^{i k x \cdot d}$ with fixed $k \in \mathbb{R}_{+}$and $d \in \mathbb{S}^{N-1}$. Then we introduce the following real-valued index function $I_{1}(z)$ for $z \in \mathbb{R}^{N}$ :

$$
I_{1}(z):=\frac{1}{\left\|a\left(\hat{x} ; \Omega^{s, t}\right)\right\|_{L^{2}\left(\mathbb{S}^{N-1}\right)}^{2}} \sum_{n=0}^{1} \sum_{p=-n}^{n}\left|\left\langle a\left(\hat{x} ; \Omega^{s, t}\right), e^{i k(d-\hat{x}) \cdot z} Y_{n}^{p}(\hat{x})\right\rangle_{L^{2}\left(\mathbb{S}^{N-1}\right)}\right|^{2},
$$

where $\langle u, v\rangle_{L^{2}\left(\mathbb{S}^{N-1}\right)}=\int_{\mathbb{S}^{N-1}} u \cdot \bar{v} d s(\hat{x})$. In $(2.9), Y_{n}^{p}(\hat{x})$ for $n \in \mathbb{N} \cup\{0\}$ and $p=-n, \ldots, n$ are the spherical harmonics which form a complete orthonormal system in $L^{2}\left(\mathbb{S}^{N-1}\right)$ (cf. [22]). It is emphasized that there is no harmonic function $Y_{1}^{0}(\hat{x})$ in the two-dimensional case, so it should be removed from the summation in (2.9) in defining $I_{1}(z)$. The next theorem about the indicating behavior of $I_{1}(z)$ is the crux of developing our Scheme I.

Theorem 2.1. Let $\Omega^{s, t}$ and $I_{1}(z)$ be described as in (2.6) and (2.9) respectively. Set

$$
M_{j}:=\frac{\left\|a\left(\hat{x} ; \Omega_{j}^{s, t}\right)\right\|_{L^{2}\left(\mathbb{S}^{N-1}\right)}^{2}}{\left\|a\left(\hat{x} ; \Omega^{s, t}\right)\right\|_{L^{2}\left(\mathbb{S}^{N-1}\right)}^{2}}, \quad j=1, \ldots, l_{t} .
$$

Then the following asymptotic expansion holds:

$$
M_{j}=M_{j}^{0}+\mathcal{O}\left(\frac{1}{L}+\rho^{N-2}(\ln \rho)^{N-3}\right), \quad j=1, \ldots, l_{t},
$$

where $M_{j}^{0}$ is a positive number independent of $L$ and $\rho$. Moreover, there exists an open neighborhood of $z_{j}, \operatorname{neigh}\left(z_{j}\right), 1 \leq j \leq l_{t}$, such that

$$
I_{1}(z) \leq M_{j}^{0}+\mathcal{O}\left(\frac{1}{L}+\rho^{N-2}(\ln \rho)^{N-3}\right) \quad \text { for } z \in \operatorname{neigh}\left(z_{j}\right),
$$

where the equality holds only at $z=z_{j}$. That is, $z_{j}$ is a local maximizer of $I_{1}(z)$ in neigh $\left(z_{j}\right)$.

In order to prove Theorem 2.1, we first present two crucial lemmas.

Lemma 2.1. Let $\Omega^{s, t}$ and $a\left(\hat{x} ; \Omega^{s, t}\right)$ be given in (2.6) and (2.8) respectively. Then it holds that

$$
a\left(\hat{x} ; \Omega^{s, t}\right)=a\left(\hat{x} ; \bigcup_{j=1}^{l_{t}} \Omega_{j}^{s, t}\right)=a\left(\hat{x} ; \bigcup_{j=1}^{l_{t}}\left(z_{j}+\rho D_{j}^{t}\right)\right)=\sum_{j=1}^{l_{t}} e^{i k(d-\hat{x}) \cdot z} a\left(\hat{x} ; \rho D_{j}^{t}\right)+\mathcal{O}\left(\frac{1}{L}\right) .
$$


Proof. First, one has

$$
a\left(\hat{x} ; \Omega^{s, t}\right)=a\left(\hat{x} ; \bigcup_{j=1}^{l_{t}}\left(z_{j}+\rho D_{j}^{t}\right)\right)=\sum_{j=1}^{l_{t}} a\left(\hat{x} ; z_{j}+\rho D_{j}^{t}\right)+\mathcal{O}\left(\frac{1}{L}\right),
$$

which was proved in [33] when $\Omega^{s, t}$ is a sound-soft obstacle. Following a similar argument, one can demonstrate (2.13) when $\Omega^{s, t}$ is a sound-hard or an impedance obstacle, or an inhomogeneous medium. On the other hand, it is straightforward to verify that

$$
a_{k}\left(\hat{x}, d ; z_{j}+\rho D_{j}^{t}\right)=e^{i k(d-\hat{x}) \cdot z_{j}} a_{k}\left(\hat{x}, d ; \rho D_{j}^{t}\right),
$$

which together with (2.13) readily gives (2.12).

The results in the following lemma can be found in $[12,23,24,29,34]$.

Lemma 2.2. Let $D$ be a bounded simply-connected $C^{2}$ domain containing the origin and $\rho D^{t}$ be a scatterer of type $t=s, h, i$ or $m$, as described in (2.1)-(2.5). Then there exists $\rho_{0} \in \mathbb{R}_{+}$ such that for $\rho<\rho_{0}$,

$$
a_{k}\left(\hat{x}, d ; \rho D^{t}\right)=c_{0} E(\rho) Y_{0}^{0}(\hat{x})+\mathcal{O}\left(\rho^{N-2}(\ln \rho)^{N-3} E(\rho)\right), \quad t=s, i \text { or } m,
$$

where $c_{0}$ is constant depending only on $D, k, d$ and $t$, but independent of $\rho$. In (2.14),

$$
E(\rho):=\rho^{N-2}(\ln \rho)^{N-3} \text { when } t=s ; \quad \rho^{N-1} \text { when } t=i ; \quad \rho^{N} \text { when } t=m .
$$

In the case when $t=h$, we have

$$
a_{k}\left(\hat{x}, d ; \rho D^{h}\right)=\rho^{N} \sum_{n=0}^{1} \sum_{p=-n}^{n} c_{n}^{p} Y_{n}^{p}(\hat{x})+\mathcal{O}\left(\rho^{N+1}\right),
$$

where the coefficients $c_{n}^{p}$ are constants depending only on $D, k, d$, but independent of $\rho$, and $Y_{1}^{0}(\hat{x})$ should be removed from the summation in (2.15) in the two-dimensional case.

Proof of Theorem 2.1. We first consider the three-dimensional sound-hard case. By Lemmas 2.1 and 2.2 we can easily see that

$$
\begin{aligned}
& a\left(\hat{x} ; \Omega^{s, h}\right)=\sum_{j=1}^{l} a\left(\hat{x} ; \Omega_{j}^{s, h}\right)+\mathcal{O}\left(\frac{1}{L}\right) \\
= & \sum_{j=1}^{l_{h}} e^{i k(d-\hat{x}) \cdot z_{j}}\left[\rho^{3} \sum_{n=0}^{1} \sum_{p=-n}^{n} c_{n, j}^{p} Y_{n}^{p}(\hat{x})+\mathcal{O}\left(\rho^{4}\right)\right]+\mathcal{O}\left(\frac{1}{L}\right) .
\end{aligned}
$$

Next, without loss of generality, we only consider the indicating behavior of $I_{1}(z)$ in $B_{\rho}\left(z_{1}\right)$, a ball of radius $\rho$ centered at $z_{1}$. Clearly, we have

$$
\left|z_{j}-z\right| \geq L \gg 1 \quad \text { for } z \in B_{\rho}\left(z_{1}\right) \text { and } j=2,3, \ldots, l_{h} .
$$


Hence, by using (2.16) and (2.17) one can show by direct calculations that

$$
\begin{aligned}
& \left|\left\langle a\left(\hat{x} ; \Omega^{s, t}\right), e^{i k(d-\hat{x}) \cdot z} Y_{n^{\prime}}^{p^{\prime}}(\hat{x})\right\rangle_{L^{2}\left(\mathbb{S}^{2}\right)}\right| \\
= & \rho^{3}\left|\left\langle e^{i k(d-\hat{x}) \cdot z_{1}} \sum_{n=0}^{1} \sum_{p=-n}^{n} c_{n, 1}^{p} Y_{n}^{p}(\hat{x}), e^{i k(d-\hat{x}) \cdot z} Y_{n^{\prime}}^{p^{\prime}}(\hat{x})\right\rangle_{L^{2}\left(\mathbb{S}^{2}\right)}+\mathcal{O}\left(\frac{1}{L}+\rho\right)\right| \\
\leq & \rho^{3}\left(\left|c_{n^{\prime}}^{p^{\prime}}\right|+\mathcal{O}\left(\frac{1}{L}+\rho\right)\right) \text { for } z \in B_{\rho}\left(z_{1}\right), \quad n^{\prime}=0,1, q^{\prime}=-n^{\prime}, \ldots, n^{\prime},
\end{aligned}
$$

where we have used (2.17) and the decaying property of oscillatory integrals for the equality relation, and the orthogonality of spherical harmonics and the Cauchy-Schwartz inequality for the inequality relation. Furthermore, due to the Cauchy-Schwartz inequality, one can verify that the equality in the last estimate of (2.18) holds only at $z=z_{1}$. On the other hand, we have

$$
\left\|a\left(\hat{x} ; \Omega^{s, t}\right)\right\|_{L^{2}\left(\mathbb{S}^{2}\right)}^{2}=\rho^{6} \sum_{j=1}^{l}\left(\sum_{n=0}^{1} \sum_{p=-n}^{n}\left|c_{n, j}^{p}\right|^{2}+\mathcal{O}\left(\frac{1}{L}+\rho\right)\right) .
$$

By (2.18)-(2.19), it is straightforward to verify the statement of the theorem by taking

$$
M_{1}^{0}=\frac{\sum_{n=0}^{1} \sum_{p=-n}^{n}\left|c_{n, 1}^{p}\right|^{2}}{\sum_{j=1}^{l} \sum_{n=0}^{1} \sum_{p=-n}^{n}\left|c_{n, j}^{p}\right|^{2}} .
$$

The other cases with $t=s, i$ and $m$ can be proved by following a similar argument to the above case with $t=h$, and using Lemmas 2.1 and 2.2.

Using Theorem 2.1, we are now ready to formulate our first imaging scheme of locating multiple small scatterer components.

\section{Scheme I}

1) For an unknown scatterer $\Omega^{s, t}$ in (2.6), collect the far-field data by sending a single incident plane wave $e^{i k x \cdot d}$ with fixed $k$ and $d$.

2) Select a sampling region with a mesh $\mathcal{T}_{h}$ containing $\Omega^{s, t}$.

3) For each sampling point $z \in \mathcal{T}_{h}$, compute the index value $I_{1}(z)$.

4) Locate all the significant local maxima of $I_{1}(z)$ on $\mathcal{T}_{h}$, which represent the locations of the scatterer components.

Remark 2.1. As it can be seen from Theorem 2.1, the indicating behavior of $I_{1}(z)$ is independent of the type of the underlying scatterer, which can be obstacle or inhomogeneous medium. Indeed, Scheme I can be extended to a much more general situation where the underlying scatterer $\Omega^{s}$ might be composed of mixed-type scatterers from different $\Omega^{s, t}$ for $t \in\{s, h, i, m\}$. We illustrate the situation by taking a special example, say, $\Omega^{s}$ consists of two components, a sound-soft 
$\Omega_{1}^{s}$ and a sound-hard $\Omega_{2}^{s}$ in three dimensions. Suppose that the relative sizes of $\Omega_{1}^{s}$ and $\Omega_{2}^{s}$ are, respectively, $\rho_{1}$ and $\rho_{2}$. According to Lemma 2.2, the scattering strength due to $\Omega_{1}^{s}$ is of order $\rho_{1}$ whereas that due to $\Omega_{2}^{s}$ is of order $\rho_{2}^{3}$. If $\rho_{1} \approx \rho_{2}$, then $\rho_{2}^{3} \ll \rho_{1}$, hence the scattering information from the sound-hard component is annihilated in the scattering data due to the sound-soft component. In this case, one cannot expect a reasonable locating by using Scheme I. However, if $\rho_{2}^{3} \sim \rho_{1}$, then it is straightforward to verify that the local maximum behavior in Theorem 2.1 holds for the locations of the two mixed-type scatterer components, hence Scheme I works to locate both of them. This observation holds for the general case with multiple scatterer components of different type. As long as the scattering strengths from different components are comparable, Scheme I is effective to locate all of them.

Remark 2.2. In [43], an orthogonality sampling method was proposed where the indicator function is related to our indicator function $I_{1}(z)$ in $(2.9)$. Indeed, if only a single far-field measurement is used, the indicator function in [43] actually corresponds to the first term in the sum defining $I_{1}(z)$ in (2.9). It can be easily verified from Lemma 2.2 and the proof of Theorem 2.1, if the underlying scatterer is sound-soft, then the two indicator functions would produce the same local maximum behavior. However, if one wants to produce an indicator function for locating a small-size scatterer independent of the physical properties of its components as discussed in the previous remark, then both the acoustic monopole and dipoles should be involved. As the orthogonality sampling method and topological derivative-based approaches (cf. [12] and [25]) are closely relate, similar observations can be made for the latter approaches.

\subsection{Numerical experiments}

In this subsection, we present some numerical tests to verify the applicability of Scheme I in both two and three dimensions. In all the tests, the exact far-field data are obtained by solving the forward equation (1.2) or (1.5) using the quadratic finite elements on a truncated circular (2D) or spherical (3D) domain enclosed by a PML layer. The forward equation is solved on a sequence of successively refined meshes till the relative error of two successive finite element solutions between the two adjacent meshes is below $0.1 \%$. Then the scattered data are transformed into the far-field data by employing the Kirchhoff integral formula on a closed circle (2D) or surface (3D) enclosing the scatterer. For scatterers of small size, we always add to the exact far-field data a uniform noise of $5 \%$ and use it as the measurement data in our numerical tests.

Example 1. The true scatterer consists of three components, a sound-soft square component (in red) with side length 0.2 located at $(-1,2)$, a sound-hard circular component (in white) with radius 0.5 located at $(1.5,0)$, and a medium square component (in yellow) with side length 0.2 located at $(-2,-1.5)$. They are respectively shown in Fig. 1(a).

We set the wave number $k$ to be 3 and choose the incident direction $d=(1,0)$, namely from left to right. The detecting wave length is larger than the sizes of all the components. Fig. 2(a) shows the indicating behavior using the indicator function (2.9) of Scheme I, and the three components of the unknown scatterer are located very well using a single detecting plane wave field. By further increasing the wave number $k$ to be 6 and adopting a different incident direction $d=(0,1)$, namely from bottom to top, we find that each component of the scatterer is highlighted as a local maximum as shown in Fig. 2(b). It is pointed out that Scheme I applies to such a complex scenario with scatterer components of mixed types when the scattering of each component are comparable, which implies that the size of the sound-hard component should be relatively larger than those of its sound-soft and medium ones in light of Remark 2.1. 


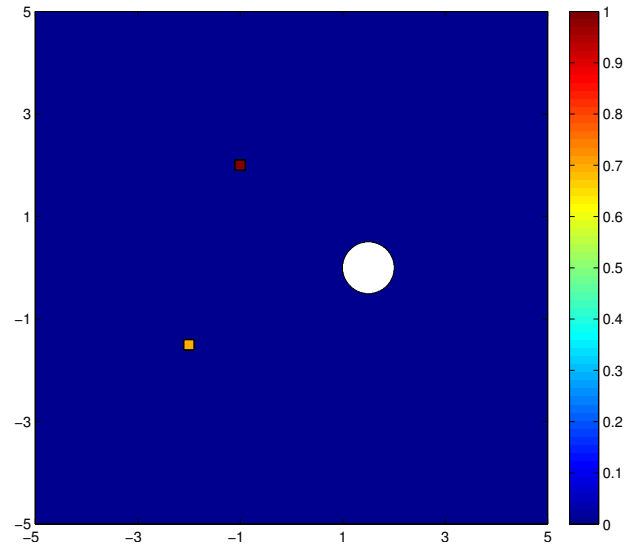

(a)

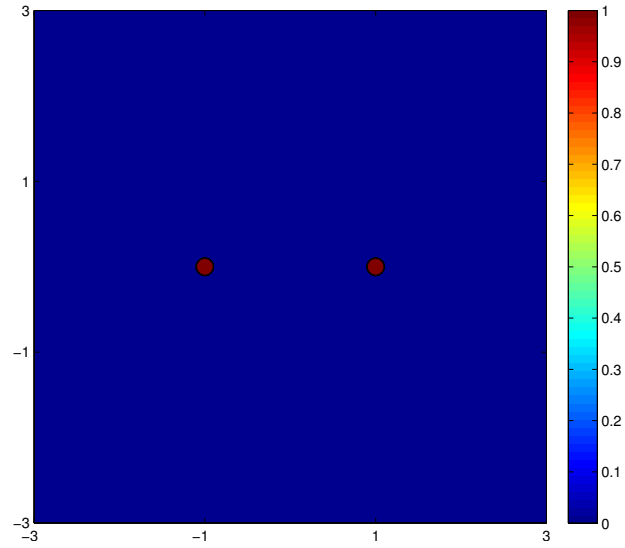

(b)

Figure 1: True scatterer components in (a) Example 1 and (b) Example 2.

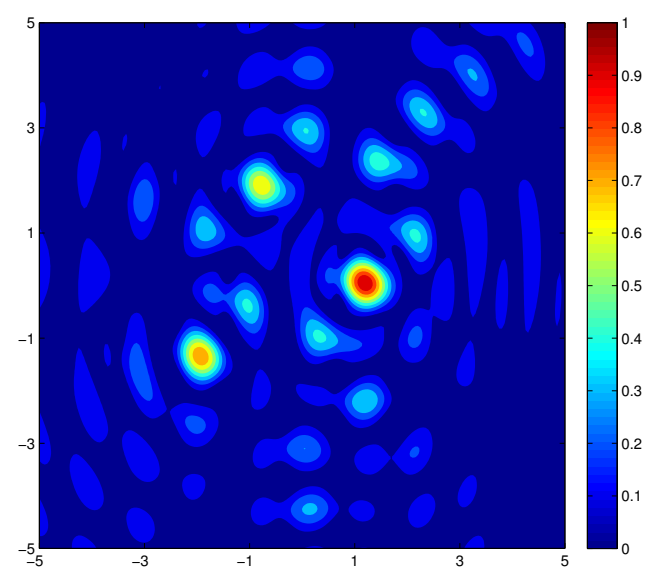

(a) $k=3, d=(1,0)$

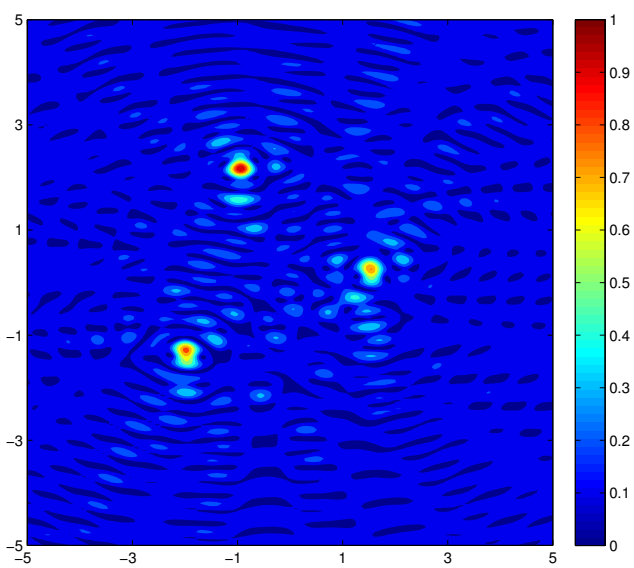

(b) $k=6, d=(0,1)$

Figure 2: Imaging of the scatterer components in Example 1 by Scheme I. 


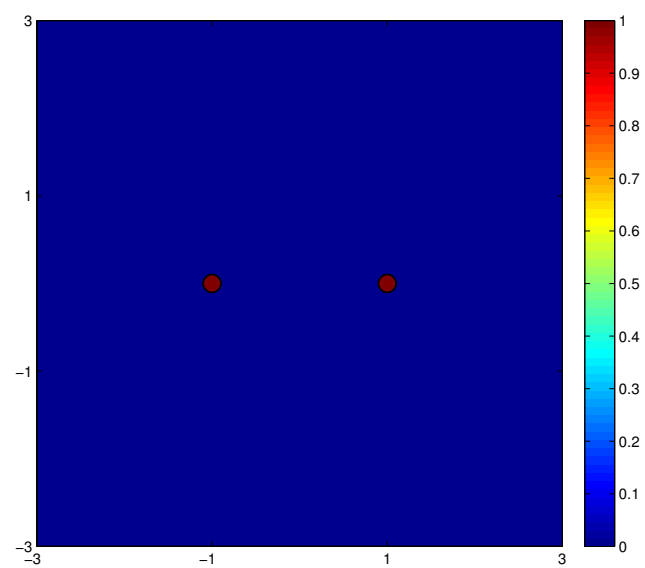

Figure 3: True scatterer components in Example 2.

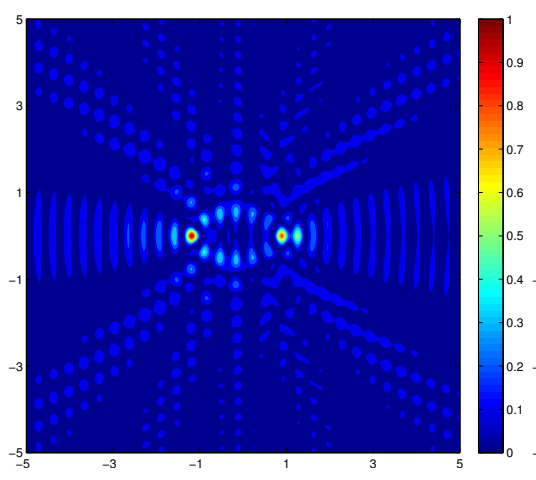

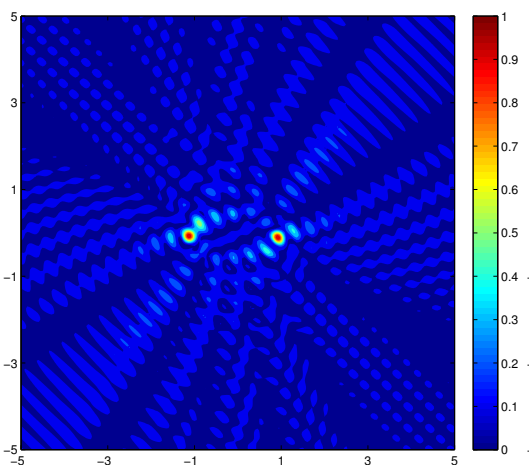

$k=9, d=(1,1)$

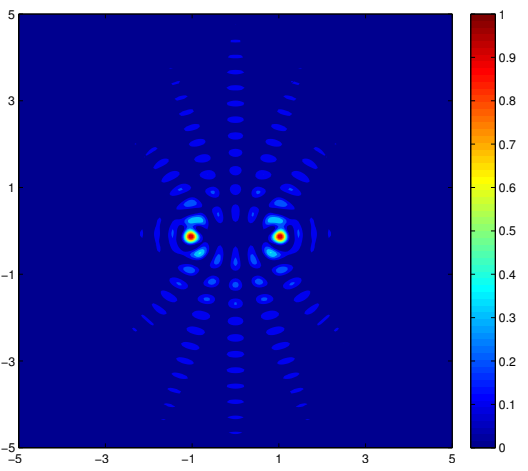

$k=9, d=(0,1)$

Figure 4: Imaging of scatterer components in Example 2 by Scheme I.

Example 2. The true scatterer consists of two sound-hard circular disks of radius 0.1, located at $(-1,0),(1,0)$ as shown in Fig. $1(\mathrm{~b})$.

Through this example, we show that Scheme I is totally independent of incident directions. It is found that we can always locate this pair of scatterer components with only one measurement data from an arbitrary incident direction. For instance, we show the cases when $k=9$ but $d=(1,0),(1,1)$ and $(0,1)$ in Fig. 4.

Example 3. We try further a complex scatterer in 3D. The wave number of the incident wave field is set to be $k=5$. The true scatterer (see Fig. 5(a)) consists of three components, namely a sound-soft cube (in red) with side length 0.2 centered at $(-1,0,1.5)$, a sound-hard sphere (in green) with radius 0.2 centered at $(2,0,0)$, and a medium cube (in blue) with side length 0.1 centered at $(-1.5,0,-1.5)$.

The resulting indicator function value distribution is plotted on a pair of orthogonal slice planes $x=0$ and $y=0$ in Fig. 5(b). As one can see, three scatterer components are well located, and their positions are visualized in the highlighted part (local maxima). Clearly, the positions of the respective detected components match quite well with the ones of the exact components.

In summary, we have observed from Examples 1-3 that Scheme I is able to locate multiple small scatterer components of an unknown number robustly and efficiently. 


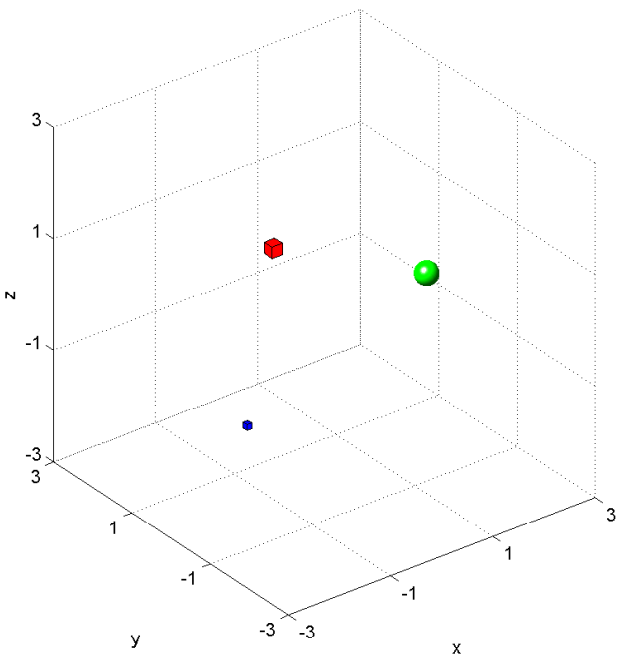

(a)

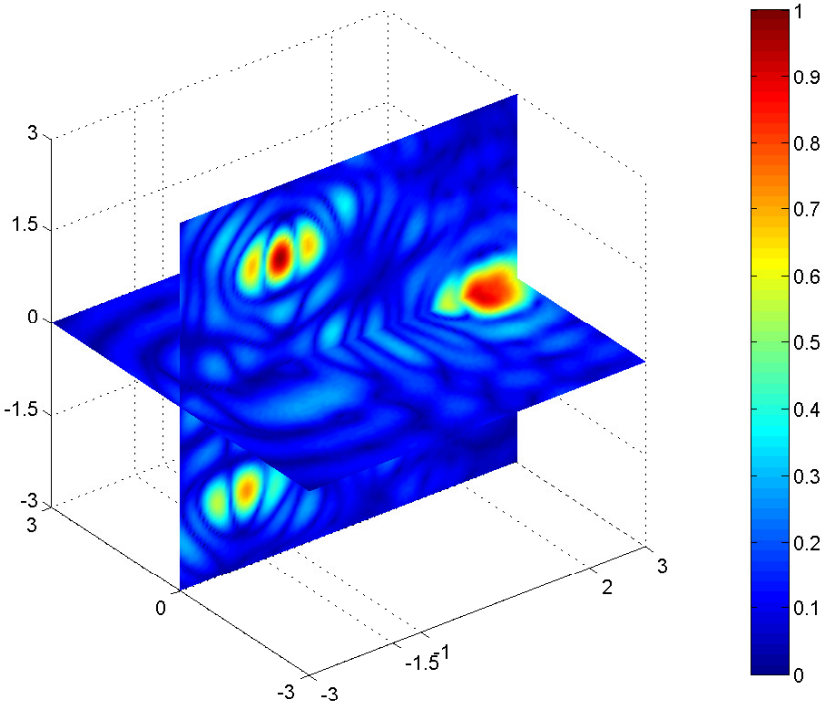

(b)

Figure 5: (a) True scatterer components in Example 3. (b) Imaging of the scatterer components in Example 3 by Scheme I.

\section{Locating scatterers of regular size}

\subsection{Scheme II}

In this section, we consider the locating of multiple scatterers of regular size. We first fix some notations that shall be used throughout the rest of the paper. Let $G$ be a bounded simplyconnected Lipschitz domain in $\mathbb{R}^{n}$ containing the origin, and $U \in S O(N)$ a rotation matrix in $\mathbb{R}^{N}$, and we define

$$
\Pi_{U} G:=U G=\{U x \mid x \in G\} .
$$

We also introduce the scaling operator as follows: for any $r \in \mathbb{R}_{+}$,

$$
\Lambda_{r} G:=r G=\{r x \mid x \in G\} .
$$

For any domain $\Omega$ of the form $\Omega:=z+\Lambda_{r} \Pi_{U} G$, we shall write the quartette $\Omega:=(G ; z, r, U)$, and call $z, r$ and $U$ respectively the location, the scale and the orientation of $\Omega$ relative to $G$. In our subsequent study, $G$ could be a sound-soft, sound-hard or impedance-type obstacle, or an inhomogeneous medium, and we shall write correspondingly $G^{t}$ with $t=s, h, i$ or $m$, and $\Omega^{t}=\left(G^{t} ; z, r, U\right)$. For the ease of exposition, we always assume that the corresponding surface impedance $\lambda$ or the refractive index $n$ is a constant in the case when $G$ is an impedance obstacle or a medium. Next, we let $\theta \in \mathbb{R}_{+}$such that $\theta \ll 1$, and $\mathscr{T}_{1}$ be a suitably chosen finite index set, such that $\left\{U_{j}\right\}_{j \in \mathscr{T}_{1}}$ be a $\theta$-net of $S O(N)$. That is, for any rotation matrix $U \in S O(N)$, there exists $j \in \mathscr{T}_{1}$ such that $\left\|U_{j}-U\right\| \leq \theta$. We define

$$
\Pi_{\theta} G:=\left\{\Pi_{U_{j}} G\right\}_{j \in \mathscr{T}_{1}} .
$$


In a similar manner, for $\Lambda_{r}$ with $r \in\left[R_{0}, R_{1}\right]$, we let $\tau \ll 1$ and $\mathscr{T}_{2}$ be a suitably chosen finite index set such that $\left\{r_{j}\right\}_{j \in \mathscr{T}_{2}}$ be an $\tau$-net of $\left[R_{0}, R_{1}\right]$. Define,

$$
\Lambda_{\tau} G=\left\{\Lambda_{j} G\right\}_{j \in \mathscr{T}_{2}} .
$$

With the above preparations, we are now ready to introduce the multiple scatterers of regular size for our subsequent study. Let $l_{t} \in \mathbb{N} \cup\{0\}, t=s, h, i$ or $m$, and $G_{j}, j \in \mathbb{N}$, be a bounded simply-connected Lipschitz domain in $\mathbb{R}^{n}$ containing the origin. We write

$$
\begin{aligned}
\mathscr{A}_{t} & =\left\{G_{j}^{t}\right\}_{j=1}^{l_{t}}, t=s, h ; \quad \mathscr{A}_{i}=\left\{G_{j}^{i}\right\}_{j=1}^{l_{i}}=\left\{\left(G_{j}^{i}, \lambda_{j}\right)\right\}_{j=1}^{l_{i}} ; \\
\mathscr{A}_{m} & =\left\{G_{j}^{m}\right\}_{j=1}^{l_{m}}=\left\{\left(G_{j}^{m}, n_{j}\right)\right\}_{j=1}^{l_{m}} ; \mathscr{A}=\bigcup_{t=s, h, i, m} \mathscr{A}_{t}:=\left\{G_{j}\right\}_{j=1}^{l^{\prime}:=l_{s}+l_{h}+l_{i}+l_{m}} .
\end{aligned}
$$

Let $l \in \mathbb{N}$ and set

$$
\Omega^{r}=\bigcup_{j=1}^{l} \Omega_{j}^{r}, \quad \Omega_{j}^{r}:=\left(\Sigma_{j} ; z_{j}, r_{j}, U_{j}\right) \quad \text { with } \Sigma_{j} \in \mathscr{A}, \quad j=1, \ldots, l .
$$

For $\Omega^{r}$ introduced in (3.7), we assume that

$$
r_{j} \in\left[R_{0}, R_{1}\right], \quad R_{0} \approx 1, \quad R_{1} \approx 1, \quad \text { and } \quad L=\min _{1 \leq j, j^{\prime} \leq l, j \neq j^{\prime}} \operatorname{dist}\left(z_{j}, z_{j^{\prime}}\right) \gg 1 .
$$

$\Omega^{r}$ represents the multiple scatterers of regular size in our current study, and we shall develop Scheme II to locate all the multiple components. We assume that the admissible class $\mathscr{A}$ is known in advance. In the physical situation, this means that $\Omega^{r}$ might be composed of multiple regular-size components of an unknown number, and each component could be from a different type: obstacles of different types or a medium. If the scatterer component is an obstacle (soundsoft, sound-hard or impedance type), then its shape must be from a known admissible class. Moreover, the surface impedance of an impedance-type component must also be known a priori. If the scatterer is a medium, then both its shape and content should be known from the admissible class. It is emphasized that the number of admissible class, namely $l^{\prime}$ in $\mathscr{A}$, and the number of the unknown scatterer components, namely $l$ in $\Omega^{r}$, are not necessarily the same. This means that a certain shaped admissible scatterer might appear more than once or does not appear in $\Omega^{r}$. For the regular-size multiple scatterers $\Omega^{r}$ described above, we shall develop Scheme II to determine the location, the size/scale and the orientation of each of its components, by using a single far-field measurement, i.e. $a\left(\hat{x} ; \Omega^{r}\right):=a_{k}\left(\hat{x}, d ; \Omega^{r}\right)$ with fixed $k \in \mathbb{R}_{+}$and $d \in \mathbb{S}^{N-1}$.

In order to present our Scheme II, we first augment the admissible class $\mathscr{A}$ as

$$
\widetilde{\mathscr{A}}=\Pi_{\theta} \Lambda_{\tau} \mathscr{A}=\bigcup_{j=1}^{l^{\prime}}\left\{\Pi_{\theta} \Lambda_{\tau} G_{j}\right\}:=\left\{\widetilde{G}_{j}\right\}_{j=1}^{l^{\prime \prime}} .
$$

We shall make the following two assumptions about the augmented admissible class $\widetilde{\mathscr{A}}$ :

(i) $a_{k}\left(\hat{x}, d ; \widetilde{G}_{j}\right) \neq a_{k}\left(\hat{x}, d ; \widetilde{G}_{j^{\prime}}\right)$ for $j \neq j^{\prime}$ and $1 \leq j, j^{\prime} \leq l^{\prime \prime}, \hat{x} \in \mathbb{S}^{N-1}$;

(ii) $\left\|a_{k}\left(\hat{x}, d ; \widetilde{G}_{j}\right)\right\|_{L^{2}\left(\mathbb{S}^{N-1}\right)} \geq\left\|a_{k}\left(\hat{x}, d ; \widetilde{G}_{j^{\prime}}\right)\right\|_{L^{2}\left(\mathbb{S}^{N-1}\right)}$ for $j<j^{\prime}$ and $1 \leq j, j^{\prime} \leq l^{\prime \prime}$. 
Assumption (ii) can be fulfilled by reordering if necessary. For assumption (i), we recall the following well-known conjecture in the theory of the inverse acoustic scattering problem:

$$
a_{k}(\hat{x}, d ; G)=a_{k}(\hat{x}, d ; \widetilde{G}) \text { if and only if } G=\widetilde{G},
$$

where $G$ and $\widetilde{G}$ are two obstacles, with $k$ and $d$ fixed. (3.10) states that a single far-field measurement can uniquely determine an acoustic obstacle. There is a widespread belief that (3.10) holds true, but there is only limited progress in the literature (cf. [35,37,38]). Clearly, if (3.10) holds true, and if $\widetilde{\mathscr{A}}$ contains only obstacle scatterers, assumption (ii) is always fulfilled. On the other hand, (3.10) does not holds true in general for inhomogeneous medium scatterers, hence if there are medium scatterers presented in $\widetilde{\mathscr{A}}$, assumption (ii) can not be always fulfilled. Nevertheless, since $\widetilde{\mathscr{A}}$ is known, assumption (ii) can always be verified in advance.

Now we introduce the following $l^{\prime \prime}$ indicator functions for identifying the multiple scatterers of $\Omega^{r}$ in (3.7):

$$
I_{2}^{j}(z)=\frac{\left|\left\langle a\left(\hat{x} ; \Omega^{r}\right), e^{i k(d-\hat{x}) \cdot z} a\left(\hat{x} ; \widetilde{G}_{j}\right)\right\rangle_{L^{2}\left(\mathbb{S}^{N-1}\right)}\right|}{\left\|a\left(\hat{x} ; \widetilde{G}_{j}\right)\right\|_{L^{2}\left(\mathbb{S}^{N-1}\right)}^{2}}, \quad \widetilde{G}_{j} \in \widetilde{\mathscr{A}}, \quad j=1,2, \ldots, l^{\prime \prime} .
$$

Next, we present a key theorem on the indicating behavior of these indicator functions, which forms the basis of our Scheme II.

Theorem 3.1. Suppose that $\widetilde{G}_{1} \in \widetilde{\mathscr{A}}$ is of the following form

$$
\widetilde{G}_{1}=\left(G_{j_{0}} ; r_{p_{0}}, U_{q_{0}}\right)=\Pi_{U_{q_{0}}} \Lambda_{r_{q_{0}}} G_{j_{0}}, \quad G_{j_{0}} \in \mathscr{A}, U_{q_{0}} \in \mathscr{T}_{1}, r_{p_{0}} \in \mathscr{T}_{2} .
$$

Suppose that in $\Omega^{r}$, there exists $J_{0} \subset\{1,2, \ldots, l\}$ such that for $j \in J_{0}$, the component $\Omega_{j}^{r}=$ $\left(\Sigma_{j} ; z_{j}, r_{j}, U_{j}\right)$ satisfies

$$
\text { (i) } \Sigma_{j}=G_{j_{0}} ; \quad \text { (ii) }\left\|U_{j}-U_{q_{0}}\right\| \leq \theta ; \quad \text { (iii) }\left\|r_{j}-r_{p_{0}}\right\| \leq \tau ;
$$

whereas for $j \in\{1,2, \ldots, l\} \backslash J_{0}$, at least one of the conditions in (3.12) is not fulfilled by the scatterer component $\Omega_{j}^{r}$. Then for each $z_{j}, j=1,2, \ldots, l$, there exists an open neighborhood of $z_{j}$, neigh $\left(z_{j}\right)$, such that

(i) if $j \in J_{0}$, then

$$
I_{2}^{1}(z) \leq 1+\mathcal{O}\left(\frac{1}{L}+\theta+\tau\right) \quad \forall z \in \operatorname{neigh}\left(z_{j}\right) .
$$

Moreover, the equality holds in the above relation only when $z=z_{j}$. That $i s, z_{j}$ is a local maximum point for $I_{2}^{1}(z)$.

(ii) if $j \in\{1,2, \ldots, l\} \backslash J_{0}$, then there exists $\epsilon_{0}>0$ such that

$$
I_{2}^{1}(z) \leq 1-\epsilon_{0}+\mathcal{O}\left(\frac{1}{L}+\theta+\tau\right) \quad \forall z \in \operatorname{neigh}\left(z_{j}\right) .
$$

In order to prove Theorem 3.1, we first derive a key lemma as follows. 
Lemma 3.1. Let $G$ be a bounded simply-connected domain in $\mathbb{R}^{N}$ containing the origin, which supports an acoustic scatterer $G^{t}, t=s, h, i$ or $m$. Then we have

$$
a_{k}\left(\hat{x}, d ; \Pi_{U} G^{t}\right)=a_{k}\left(U^{T} \hat{x}, U^{T} d ; G\right) \quad \text { for } U \in S O(n)
$$

and

$$
a_{k}\left(\hat{x}, d ; \Lambda_{r} G^{t}\right)=r a_{k r}(\hat{x}, d ; G) \quad \text { for } r \in \mathbb{R}_{+} .
$$

Proof. (3.15) and (3.16) can be readily verified by a change of variables in the corresponding scattering systems.

Proof of Theorem 3.1. First it follows by Lemma 2.1 that

$$
\begin{aligned}
& a_{k}\left(\hat{x}, d ; \Omega^{r}\right)=a_{k}\left(\hat{x}, d ; \bigcup_{j=1}^{l} \Omega_{j}^{r}\right)=\sum_{j=1}^{l} a_{k}\left(\hat{x}, d ; \Omega_{j}^{r}\right)+\mathcal{O}\left(\frac{1}{L}\right) \\
= & \sum_{j=1}^{l} a_{k}\left(\hat{x}, d ;\left(\Sigma_{j} ; z_{j}, r_{j}, U_{j}\right)\right)+\mathcal{O}\left(\frac{1}{L}\right) \\
= & \sum_{j=1}^{l} a_{k r_{j}}\left(U_{j}^{T} \hat{x}, U_{j}^{T} d ; \Sigma_{j}\right) e^{i k(d-\hat{x}) \cdot z_{j}}+\mathcal{O}\left(\frac{1}{L}\right) .
\end{aligned}
$$

Then by (3.12) and Lemma 3.1, we have that for $j_{0} \in J_{0}$,

$$
\begin{aligned}
a_{k r_{j}}\left(U_{j}^{T} \hat{x}, U_{j}^{T} d ; \Sigma_{j}\right) & =a_{k r_{p_{0}}}\left(\left(U_{q_{0}}\right)^{T} \hat{x},\left(U_{q_{0}}\right)^{T} d ; G_{j_{0}}\right) e^{i k(d-\hat{x}) \cdot z}+\mathcal{O}(\theta+\tau) \\
& =a_{k}\left(\hat{x}, d ; \widetilde{G}_{1}\right)+\mathcal{O}(\theta+\tau) .
\end{aligned}
$$

Hence we obtain using (3.17) and (3.18) that

$$
a\left(\hat{x} ; \Omega^{r}\right)=\sum_{j \in J_{0}} a\left(\hat{x} ; \widetilde{G}_{1}\right) e^{i k(d-\hat{x}) \cdot z_{j}}+\sum_{j \in\{1, \ldots, l\} \backslash J_{0}} a\left(\hat{x} ; \Omega_{j}^{r}\right)+\mathcal{O}\left(\frac{1}{L}+\theta+\tau\right) .
$$

For $j_{0} \in J_{0}$, by (3.19) we can show that for $z \in \operatorname{neigh}\left(z_{j_{0}}\right)$,

$$
\begin{aligned}
& \left|\left\langle a\left(\hat{x} ; \Omega^{r}\right), e^{k(d-\hat{x}) \cdot z} a\left(\hat{x} ; \widetilde{G}_{1}\right)\right\rangle_{L^{2}\left(\mathbb{S}^{N-1}\right)}\right| \\
= & \left|\left\langle a\left(\hat{x} ; \widetilde{G}_{1}\right) e^{i k(d-\hat{x}) \cdot z_{j}}, e^{i k(d-\hat{x}) \cdot z} a\left(\hat{x} ; \widetilde{G}_{1}\right)\right\rangle_{L^{2}\left(\mathbb{S}^{N-1}\right)}\right|+\mathcal{O}\left(\frac{1}{L}+\theta+\tau\right) \\
\leq & \left\|a\left(\hat{x} ; \widetilde{G}_{1}\right)\right\|_{L^{2}\left(\mathbb{S}^{N-1}\right)}^{2}+\mathcal{O}\left(\frac{1}{L}+\theta+\tau\right) .
\end{aligned}
$$

For the equality relation in (3.20), we have made use of the Riemann-Lebesgue Lemma about oscillatory integrals by noting $\left|z_{j}-z\right| \geq L \gg 1$ for $1 \leq j \leq l, j \neq j_{0}$ and $z \in \operatorname{neigh}\left(z_{j_{0}}\right)$ by means of (3.8). For the last relation in (3.20), we have applied the Cauchy-Schwartz inequality, and it is easily seen that the equality holds only at $z=z_{j_{0}}$. These observations clearly imply 
(3.13) for $z \in \operatorname{neigh}\left(z_{j_{0}}\right)$ and $j_{0} \in J_{0}$. On the other hand, by a similar argument, together with assumption (i) on $\widetilde{\mathscr{A}}$, we can directly verify that

$$
I_{2}^{1}\left(z_{j}\right)<1+\mathcal{O}\left(\frac{1}{L}+\theta+\tau\right), \quad j \in\{1,2, \ldots, l\} \backslash J_{0},
$$

which readily implies (3.14).

Based on Theorem 3.1, we are now ready to formulate Scheme II for locating the multiple scatterer components of regular size in $\Omega^{r}$ successively.

\section{Scheme II}

1) For the admissible scatterer class $\mathscr{A}$ in (3.6), formulate the augmented admissible class $\widetilde{\mathscr{A}}$ as that given in (3.9).

2) Collect in advance the far-field patterns associated with the admissible reference scatterer space $\widetilde{\mathscr{A}}$ corresponding to a single incident plane wave $e^{i k x \cdot d}$ with fixed $k$ and $d$, and reorder $\mathscr{A}$ if necessary so that assumptions (i) and (ii) are satisfied.

3) For an unknown scatterer $\Omega^{r}$ in (3.7), collect the far-field data corresponding to the single incident plane wave as specified in 2).

4) Select a sampling region with a mesh $\mathcal{T}_{h}$ containing $\Omega^{r}$.

5) Set $j=1$.

6) For each sampling point $z \in \mathcal{T}_{h}$, compute the index value $I_{2}^{j}(z)$.

7) Locate all those significant local maxima of $I_{2}^{j}(z)$ satisfying $I_{2}^{j}(z) \approx 1$ for the scatterer components of the form $z+\widetilde{G}_{j}$.

8) Remove all the sampling points inside those $N_{i d}$ identified components $z+\widetilde{G}_{j}$ found in 7 ) from $\mathcal{T}_{h}$. Subtract the individual far-field patterns associated with those already reconstructed components in 7) and their respective identified locations $z_{j}$ from the far field as follows:

$$
a\left(\hat{x} ; \Omega^{r}\right):=a\left(\hat{x} ; \Omega^{r}\right)-\sum_{j=1}^{N_{i d}} e^{i k(d-\hat{x}) \cdot z_{j}} a\left(\hat{x} ; \widetilde{G}_{j}\right)
$$

Note that the updated far-field pattern in $(3.22)$ is still denoted by $a\left(\hat{x} ; \Omega^{r}\right)$, which will be further checked by subsequent reference components in (3.11).

9) If $j=l^{\prime \prime}$, i.e., the maximum number of the unknown component reaches, then stop the reconstruction; otherwise, set $j:=j+1$, and go to 6 ). 


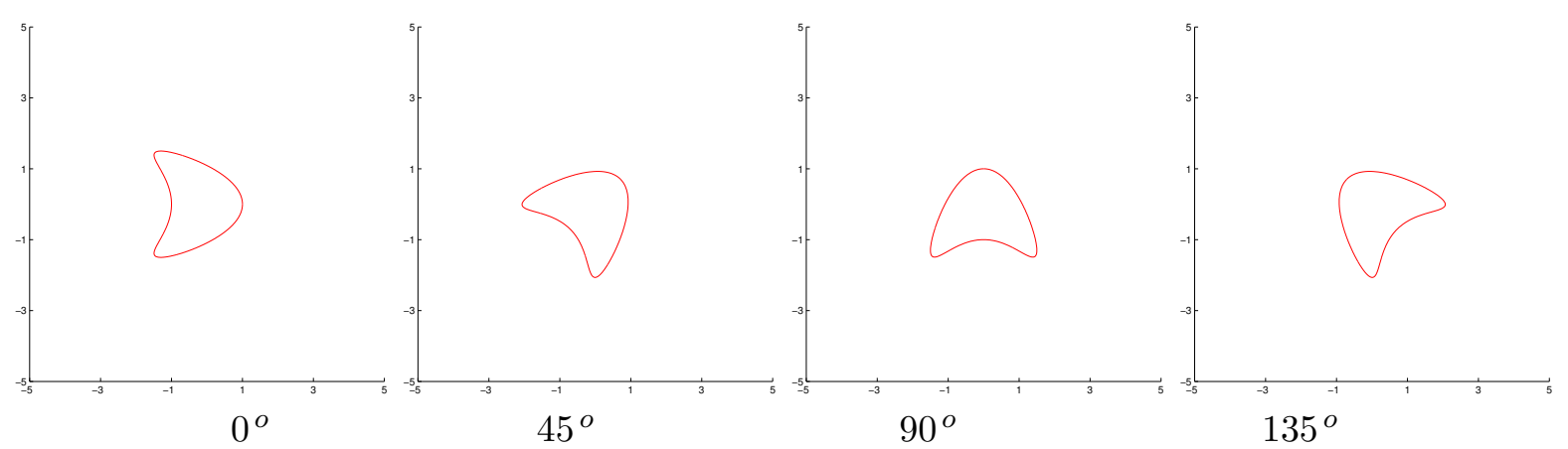

Figure 6: Basic scatterer components : a reference kite with first four orientations.

Remark 3.1. In (3.7), it is assumed that the admissible class $\mathscr{A}$ contains exactly the base scatterer $\Sigma_{j}$ of the unknown scatterer component $\Omega_{j}^{r}$ in $\Omega^{r}$. However, our Scheme II relies on the augmented admissible class $\widetilde{\mathscr{A}}$, which may contain only an approximate scatterer configuration to the target scatterer component $\Omega_{j}^{r}$ in $\Omega^{r}$. Hence, if the admissible class $\mathscr{A}$ contains only an approximate base scatterer to $\Sigma_{j}$ of the unknown scatterer component $\Omega_{j}^{r}$ in $\Omega^{r}$, Scheme II would still work, and in fact, it can be easily justified from the proof of Theorem 3.1. This point will be also illustrated by our numerical experiments in Example 5 in Subsection 3.2.

Remark 3.2. The introduction of a known admissible class $\mathscr{A}$ in our algorithm is related to the dictionary matching algorithms that have been recently investigated in a series of works by Ammari and his collaborators $[1,3,14]$, where some a priori known base shapes form a dictionary for the reconstruction. We also remark that comparable indicator functions are used in a recent work [15] for reconstructing the acoustic scatterers at small scale and regular scale, respectively.

\subsection{Numerical experiments}

We proceed now with some numerical tests using Scheme II to detect multiple scatter components of regular size. The synthetic far-field data is generated in the same manner as stated in Section 2.2 , then a uniform noise of $3 \%$ is added to the synthetic data.

Two geometries will be considered for the scatterer components in our numerical tests. They are characterized by the following $2 \mathrm{D}$ parametric curves

$$
\begin{aligned}
\text { Peanut : } & \left\{(x, y): x=\sqrt{3 \cos ^{2}(s)+1} \cos (s), y=\sqrt{3 \cos ^{2}(s)+1} \sin (s), 0 \leq s \leq 2 \pi\right\}, \\
\text { Kite : } & \{(x, y): x=\cos (s)+0.65 \cos (2 s)-0.65, y=1.5 \sin (s), 0 \leq s \leq 2 \pi\} .
\end{aligned}
$$

These will be denoted by $\mathbf{P}$ and $\mathbf{K}$, respectively, for short. The candidate data set $\widetilde{\mathscr{A}}$ includes the far-field data of both reference components $\mathbf{P}$ and $\mathbf{K}$, and is further lexicographically augmented by a collection of a priori known orientations and sizes. More precisely, the augmented data set is obtained by rotating $\mathbf{P}$ and $\mathbf{K}$ in the $x-y$ plane every 45 degrees as shown in Figs. 6 and 7 , respectively, and by scaling $\mathbf{P}$ and $\mathbf{K}$ by one half, one and twice.

For imaging of regular-size scatterers, we adopt a technique from image contrast enhancement by increasing the order of power of the indicator function $I_{2}^{j}(z)$, namely $\left(I_{2}^{j}(z)\right)^{\alpha}$ is employed as the indicator, where $\alpha$ is taken in our experiments to be 2,3 or 4 . This contrast enhancing technique helps keep the maxima around 1 and reduces significantly the potential region where multiple scattering happens. 


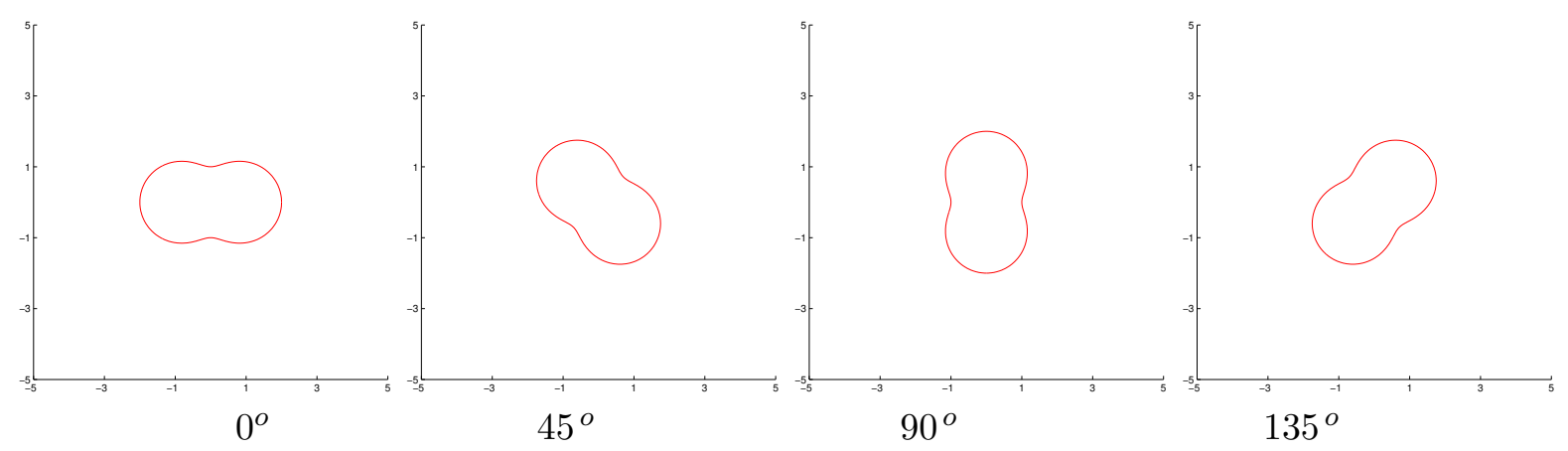

Figure 7: Basic scatterer components : a reference peanut with four orientations.

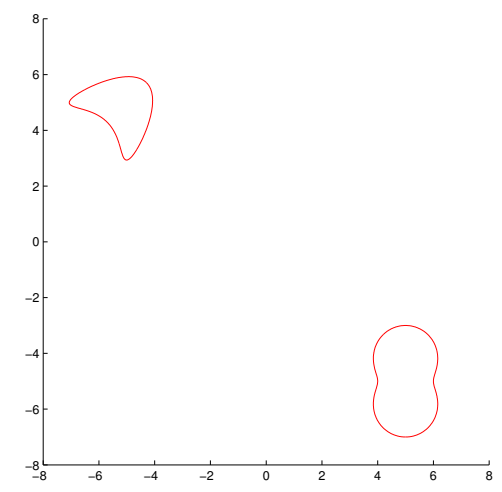

Figure 8: True scatterer components in Example 4.

As we recall, Scheme II will locate all the components, one by one, by computing an index function for each reference object in the augmented admissible class, which tells the shapes, orientations and scales of all potential components.

Example 4. The true scatterer consists of two components, a medium kite located at $(-5,5)$ and a medium peanut located at $(5,-5)$, see Fig. 8 . The wavenumber of the incident field and the incident direction are set to be $k=5$ and $d=(1,1) / \sqrt{2}$.

In the first stage, the reference peanut component is first chosen to be located, based on the reordering of the magnitudes of the far-field patterns of all the reference scatterer components. We plot in Fig. 9 the indicator function value distribution by testing reference data associated with four orientations. It clearly indicates the right position of the peanut when the orientation angle of the peanut is 90 degrees and there are a local maximum close to the unity, which implicitly gives hints about the scatterer's shape, orientation and scale by incorporating the relevant message carried in the reference data. In Fig. 9(a), we plot a superimposed image of the indicating value distribution of the four aforementioned images by taking the maximum of four indicator function values pointwise. After obtaining the position of the peanut component, it is now possible to identify the first unknown scatterer component in Fig. 9(b).

Once the peanut component is found, then we proceed by subtracting the far-field contribution of the detected peanut component from the total far-field pattern. We can then find the kite's position reasonably; see Fig. 10. We see that only the configuration with 45 degree maximizes the indicator function to achieve nearly the unity and indicates the position of the detected kite component very clearly. In Fig. 10(a), we plot a superimposed image of maximizing 


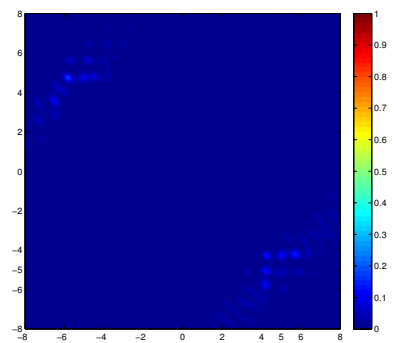

$0^{\circ}$

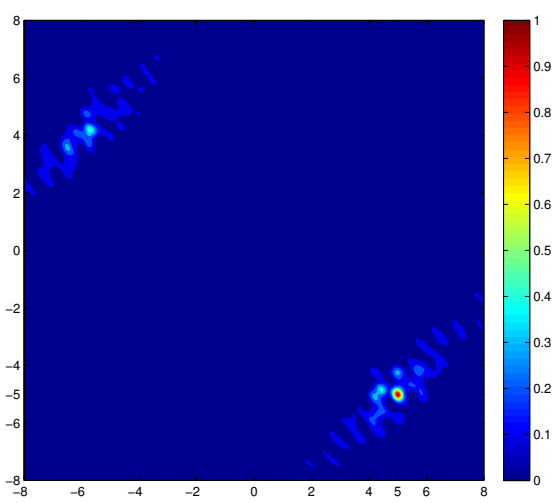

(a)

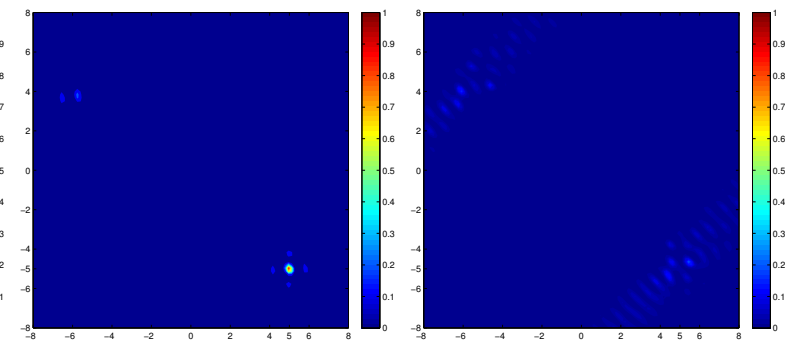

$90^{\circ}$

$135^{\circ}$

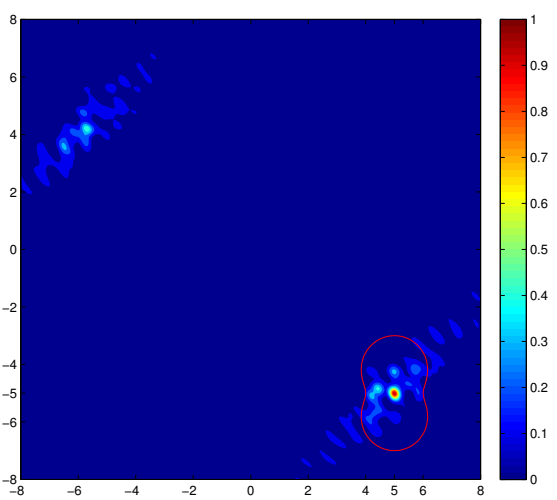

(b)

Figure 9: Example 4. From left to right in the first row: Imaging of the indicator function value distribution by testing the reference far-field data of the basis peanut $(k=5)$ at four orientations of $0,45,90$ and 135 degrees, respectively; (a) a superimposed image of the four indicating images aforementioned by taking the maximum of four indicator functions pointwise; (b) Reconstruction of the peanut component. 
the eight aforementioned image values. After obtaining the position of the kite component, it is now possible to identify the second unknown scatterer component in Fig. 9(b) by combining the information carried implicitly in the reference data, which tells us not only the shape of the scatterer but also its size and orientation.

Example 5. To further study the reliability of our locating scheme from a generic admissible reference class, we keep the admissible class unchanged but perturb pointwisely the parametric forms of the true scatterers $\mathbf{P}$ and $\mathbf{K}$ by $5 \%$ uniform noise with respect their respective centers as shown in Fig. 11. Now the admissible set is only an approximate class to describe the scatterer components.

Scheme II is repeated for such an interesting setting. The measured far-field pattern is first compared with the reference peanut data set according to (3.11). The highlighted red dot as shown in Fig. 12 when the orientation is 90 degrees tells us the rough position. Compared with Fig. 9, there exist some small ripples in the contour plots in Fig. 12 which are rather weak and do not affect the locating accuracy. Those weak ripples can be understood as some additional noise caused by the small geometric difference between the unknown components and their approximate class.

After subtracting the corresponding far-field pattern detected in the first step, we proceed with the locating of the perturbed kite components. The indicating contour plots associated with the first four orientations are shown in Figure 13. Except for some oscillating ripples, the correct position of the perturbed kite component can be well located by the red dot when the orientation is 45 degrees.

\section{Locating scatterers of multiple scales}

\subsection{Scheme III}

In this section, we consider locating multiple scatterers of multiple scales of the form

$$
\Omega^{m}=\Omega^{s, t} \cup \Omega^{r}
$$

where $\Omega^{s, t}$ is the scatterer of small size described in Section 2.1 (cf. (2.6)-(2.7)) and $\Omega^{r}$ is the scatterer of regular size described in Section 3.1 (cf. (3.7)-(3.8)). In addition to the respective assumptions on the small-scale scatterer components of $\Omega^{s, t}$ and the regular-scale scatterer components of $\Omega^{r}$ in Sections 2.1 and 3.1, we shall further assume that

$$
L=\operatorname{dist}\left(\Omega^{s, t}, \Omega^{r}\right) \gg 1 .
$$

By Lemma 2.1, one has

$$
a_{k}\left(\hat{x}, d ; \Omega^{m}\right)=a_{k}\left(\hat{x}, d ; \Omega^{s, t}\right)+a_{k}\left(\hat{x}, d ; \Omega^{r}\right)+\mathcal{O}\left(\frac{1}{L}\right) .
$$

On the other hand, by Lemma 2.2 and (2.16), one further sees that

$$
\left|a_{k}\left(\hat{x}, d ; \Omega^{s, t}\right)\right| \ll\left|a_{k}\left(\hat{x}, d ; \Omega^{r}\right)\right| .
$$

Hence we know

$$
a_{k}\left(\hat{x}, d ; \Omega^{r}\right) \approx a_{k}\left(\hat{x}, d ; \Omega^{m}\right)
$$




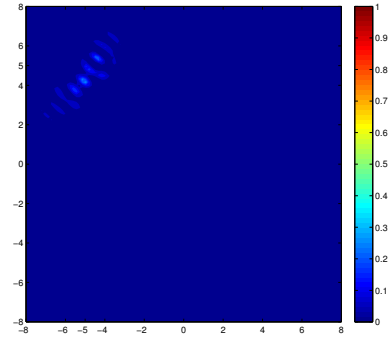

$0^{\circ}$

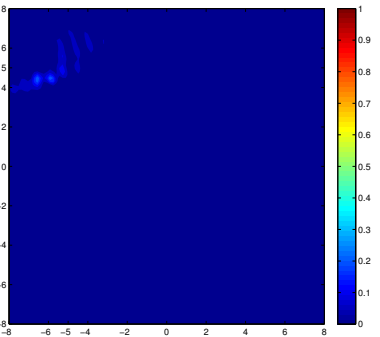

$180^{\circ}$

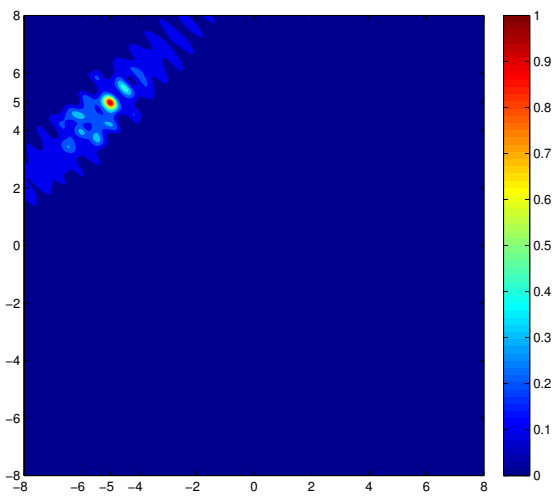

(a)

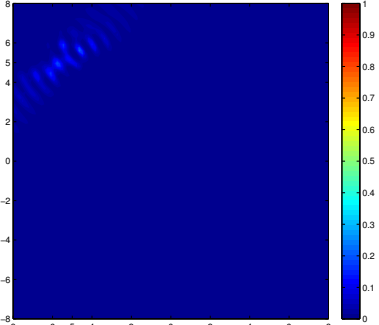

$90^{\circ}$

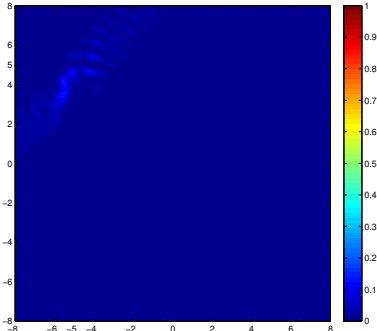

$270^{\circ}$

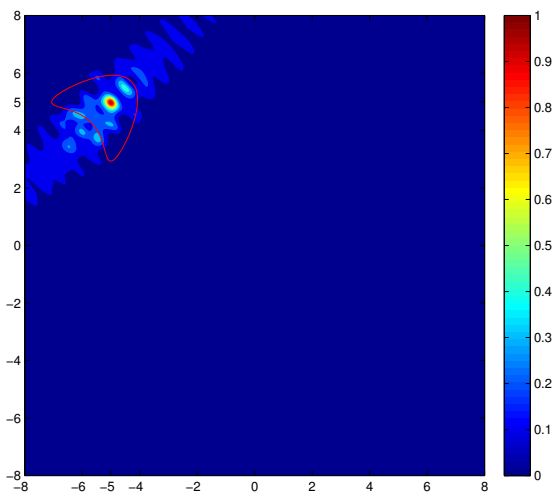

(b)

Figure 10: Example 4. From left to right in the first two rows: Imaging of the indicator function value distribution by testing the reference far-field data of the basis kite $(k=5)$ at eight orientations of $0,45,90,135,180,225,270$ and 315 degrees, respectively; (a) a superimposed image of the eight indicating images aforementioned by taking the maximum of eight indicator functions pointwise; (b) Reconstruction of the kite component. 

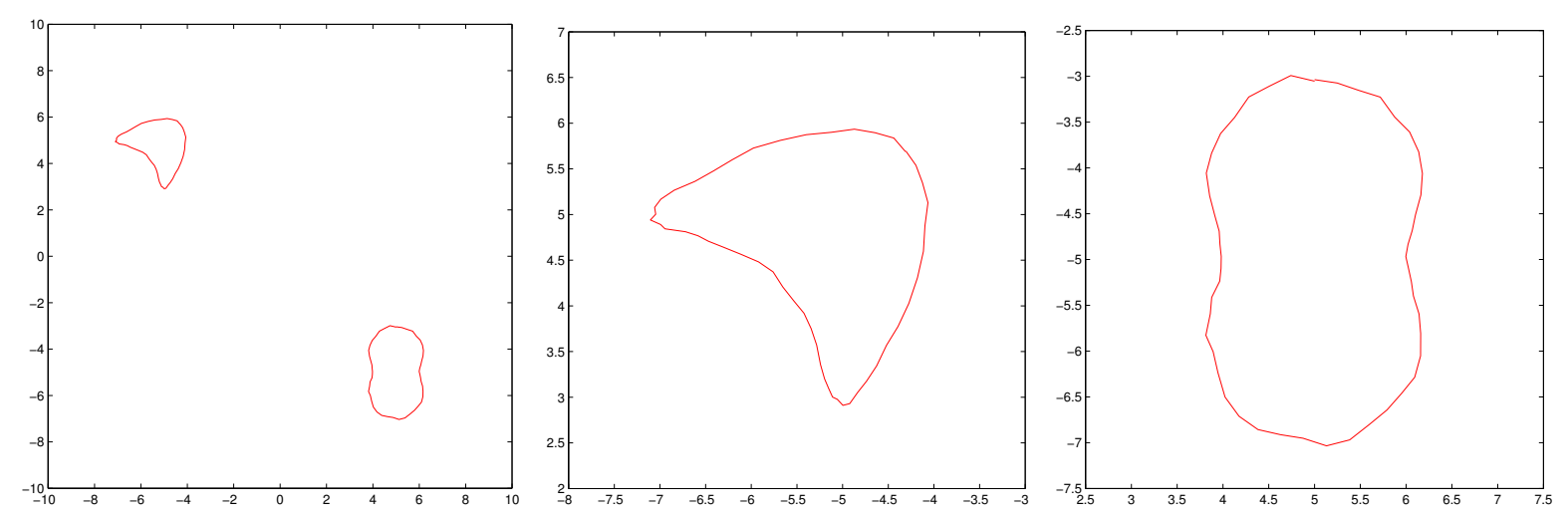

Figure 11: Perturbed scatterer components (left) and their respective zoomed-in image (middle, right) in Example 5.

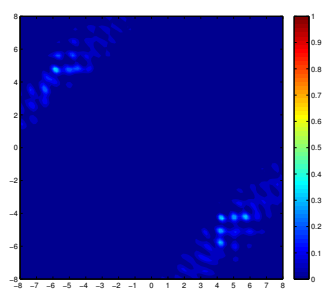

$0^{\circ}$

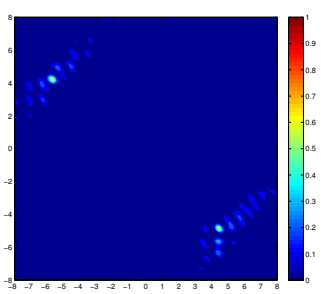

$45^{\circ}$

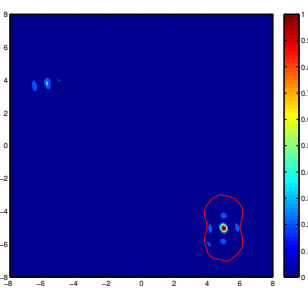

$90^{\circ}$

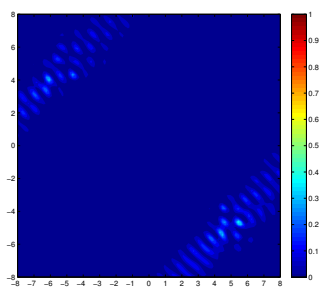

$135^{\circ}$

Figure 12: Example 5. From left to right: Imaging of the indicator function value distribution by testing the reference far-field data of the basis kite $(k=5)$ at first four orientations of 0 , 45, 90 and 135 degrees, respectively; The highlighted red dot reveals the reconstruction of the perturbed peanut component at the orientation of 90 degrees.

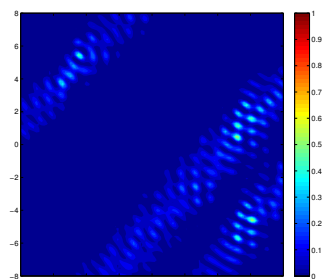

$0^{\circ}$

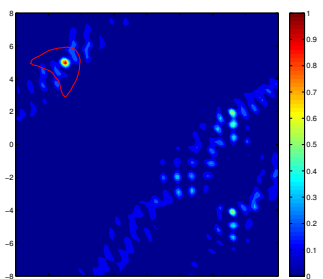

$45^{\circ}$

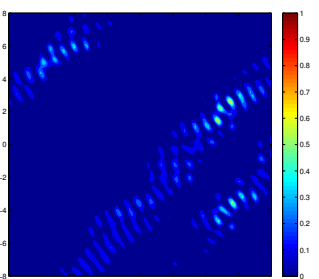

$90^{\circ}$

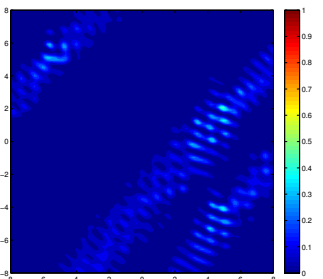

$135^{\circ}$

Figure 13: Example 5. From left to right: Imaging of the indicator function value distribution by testing the reference far-field data of the basis peanut $(k=5)$ at four orientations of 0 , 45, 90 and 135 degrees, respectively. The highlighted red dot reveals the reconstruction of the perturbed kite component at the orientation of 45 degrees.. 
Therefore, it is natural to locate the scatterers $\Omega^{m}$ of multiple scales in a two-stage manner as follows. First, by (4.5), one uses $a_{k}\left(\hat{x}, d ; \Omega^{m}\right)$ as the far-field data for Scheme II to locate the regular-scale scatterers in $\Omega^{r}$; that is, one can (approximately) find

$$
\Omega_{j}^{r}=\left(\Sigma_{j} ; z_{j}, r_{j}, U_{j}\right) \text { with } \Sigma_{j} \in \mathscr{A}, \quad j=1, \ldots, l .
$$

Second, after locating $\Omega_{j}^{r}$, one can calculate that

$$
a_{k}\left(\hat{x}, d ; \Omega^{s, t}\right) \approx a_{k}\left(\hat{x}, d ; \Omega^{m}\right)-a_{k}\left(\hat{x}, d ; \Omega^{r}\right) .
$$

Then, using the far-field data obtained above to Scheme I, one can locate the multiple smallscale scatterers in $\Omega^{s, t}$. However, we would like to emphasize the following two facts: first, the size contrast between $\Omega^{r}$ and $\Omega^{s, t}$ can not be too sharp, since otherwise by (4.4) and (4.5), the scattering information of $\Omega^{s, t}$ might be overwhelmed by that of $\Omega^{r}$; second, one should have fine reconstructions of $\Omega_{j}^{r}$ 's in the first stage. Indeed, in the first-stage reconstruction, instead of (4.6), one has

$$
\widehat{\Omega}_{j}^{r}:=\left(\Sigma_{j} ; \widehat{z}_{j}, \widehat{r}_{j}, \widehat{U}_{j}\right) \approx\left(\Sigma_{j} ; z_{j}, r_{j}, U_{j}\right), \quad \Sigma_{j} \in \mathscr{A}, \quad j=1, \ldots, l,
$$

where $\widehat{z}_{j}, \widehat{r}_{j}$ and $\widehat{U}_{j}$ are, respectively, approximations to $z_{j}, r_{j}$ and $U_{j}$. Hence, by (4.7), the far-field data used in the second stage of the reconstruction is

$$
a_{k}\left(\hat{x}, d ; \Omega^{s, t}\right) \approx a_{k}\left(\hat{x}, d ; \Omega^{m}\right)-a_{k}\left(\hat{x}, d ; \Omega^{r}\right)+\left(a_{k}\left(\hat{x}, d ; \Omega_{j}^{r}\right)-a_{k}\left(\hat{x}, d ; \widehat{\Omega}_{j}^{r}\right)\right) .
$$

If the reconstructed scatterer $\left(\Sigma_{j} ; \widehat{z}_{j}, \widehat{r}_{j}, \widehat{U}_{j}\right)$ is not close enough to the true scatterer $\left(\Sigma_{j} ; z_{j}, r_{j}, U_{j}\right)$, the error produced by $a_{k}\left(\hat{x}, d ; \Omega_{j}^{r}\right)-a_{k}\left(\hat{x}, d ; \widehat{\Omega}_{j}^{r}\right)$ would dominate over the weak scattering from $\Omega^{s, t}$. In order to overcome this error-sensitivity problem, we propose the following local tuning technique to be incorporated into the above two-stage reconstruction of $\Omega^{m}$.

Local tuning technique. Let $\left\{U_{j}\right\}_{j \in \mathscr{T}_{1}}$ and $\left\{r_{j}\right\}_{j \in \mathscr{T}_{2}}$ be the two given sets of rotations and scalings and $\mathcal{T}_{h}$ be the sampling mesh introduced in Section 3.1 , and $\left(\Sigma_{j} ; \widehat{z}_{j}, \widehat{U}_{j}, \widehat{r}_{j}\right), j=$ $1, \ldots, l$ be the reconstructed scatterers described above. For a properly chosen $\delta \in \mathbb{R}_{+}$, let $\mathcal{N}_{1}^{j}, \mathcal{N}_{2}^{j}$, and $\mathcal{N}_{3}^{j}$ be, respectively, $\delta$-neighborhoods of $\widehat{z}_{j}, \widehat{U}_{j}$ and $\widehat{r}_{j}, j=1, \ldots, l$. Then let

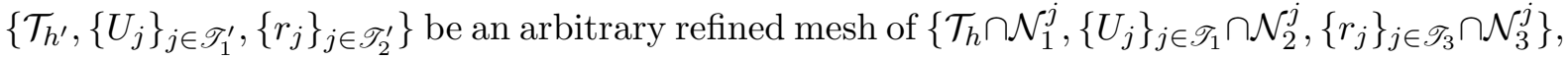
then we call

$$
\widehat{\widehat{\Omega}_{j}^{r}}:=\left(\Sigma_{j} ; \widehat{\widehat{z}}_{j}, \widehat{\widehat{r}}_{j}, \widehat{\widehat{U}}_{j}\right) \quad \text { for } \quad \widehat{\widehat{z}}_{j} \in \mathcal{T}_{h^{\prime}}, \quad \widehat{\widehat{r}}_{j} \in\left\{r_{j}\right\}_{j \in \mathscr{T}_{2}^{\prime}}, \quad \widehat{\widehat{U}}_{j} \in\left\{U_{j}\right\}_{j \in \mathscr{T}_{1}^{\prime}}
$$

a local tuneup of $\widehat{\Omega}_{j}^{r}=\left(\Sigma_{j} ; \widehat{z}_{j}, \widehat{r}_{j}, \widehat{U}_{j}\right)$ relative to $\left\{\mathcal{T}_{h^{\prime}},\left\{r_{j}\right\}_{j \in \mathscr{T}_{2}^{\prime}},\left\{U_{j}\right\}_{j \in \mathscr{T}_{1}^{\prime}}\right\}$ for $j=1,2, \ldots, l$.

Now we define

$$
\widehat{\widehat{\Omega^{r}}}:=\bigcup_{j=1}^{l} \widehat{\widehat{\Omega_{j}^{r}}},
$$

where each $\widehat{\widehat{\Omega_{j}^{r}}}$ is a local tuneup relative to $\left\{\mathcal{T}_{h^{\prime}},\left\{r_{j}\right\}_{j \in \mathscr{T}_{2}^{\prime}},\left\{U_{j}\right\}_{j \in \mathscr{T}_{1}^{\prime}}\right\}$ for $j=1,2, \ldots, l$. We call $\widehat{\widehat{\Omega^{r}}}$ a local tuneup of $\widehat{\Omega}^{r}$ relative to $\left\{\mathcal{T}_{h^{\prime}},\left\{r_{j}\right\}_{j \in \mathscr{T}_{2}^{\prime}},\left\{U_{j}\right\}_{j \in \mathscr{T}_{1}^{\prime}}\right\}$ for $j=1,2, \ldots, l$.

With the above preparations, the local tuning can be proceeded as follows: 
For each local tuneup $\widehat{\widehat{\Omega}^{r}}$, we compute

$$
\widehat{a}_{k}(\hat{x}, d):=a_{k}\left(\hat{x}, d ; \Omega^{m}\right)-a_{k}\left(\hat{x}, d ; \widehat{\widehat{\Omega^{r}}}\right),
$$

then apply the resulting far-field data to Scheme I. By running through all the local tuneups relative to $\left\{\mathcal{T}_{h^{\prime}},\left\{r_{j}\right\}_{j \in \mathscr{T}_{2}^{\prime}},\left\{U_{j}\right\}_{j \in \mathscr{T}_{1}^{\prime}}\right\}$ according to the above procedure, one can locate all the clustered local maximum points on $\mathcal{T}_{h}$, which represents the locations of the small scatterer components of $\Omega^{s, t}$.

By using the local tuning technique, one can not only locate the small-size scatterers components of $\Omega^{s, t}$, but also improve the reconstruction of the regular-size scatterers. Indeed, it can be easily seen that the local tuneup, $\widehat{\Omega^{r}}$, which is used in the local tuning that can produce the clustered local maximum points for Scheme I, is a more accurate updating of the reconstruction $\widehat{\Omega}^{r}$.

In summary, we are now ready to formulate Scheme III for locating the multiple multiscale scatterers of $\Omega^{m}$ in (4.1).

\section{Scheme III}

1) Collect a single far-field measurement $a_{k}\left(\hat{x}, d ; \Omega^{m}\right)$ corresponding to $\Omega^{m}=\Omega^{s, t} \cup \Omega^{r}$ in (4.1).

2) Select a sampling region with a mesh $\mathcal{T}_{h}$ containing $\Omega^{m}$.

3) Let $\Omega^{r}$ be given as in (3.7). Apply Scheme II with $a_{k}\left(\hat{x}, d ; \Omega^{m}\right)$ as the far-field data, and locate the rough scatterer components of $\Omega^{m}$,

$$
\widehat{\Omega}_{j}^{r}=\left(\Sigma_{j} ; \widehat{z}_{j}, \widehat{r}_{j}, \widehat{U}_{j}\right), \quad \Sigma_{j} \in \mathscr{A}, \quad j=1, \ldots, l,
$$

where $\widehat{z}_{j} \in \mathcal{T}_{h}, \widehat{r}_{j} \in\left\{r_{j}\right\}_{j \in \mathscr{T}_{2}}$ and $\widehat{U}_{j} \in\left\{U_{j}\right\}_{j \in \mathscr{T}_{1}}$ (cf. Section 3.1).

4) Apply the local tuning technique as stated below to update $\widehat{\Omega}_{j}^{r}, j=1, \ldots, l$, and locate the small-scale components of $\Omega^{s, t}$.

a) For each reconstructed $\widehat{\Omega}_{j}^{r}$ in (4.13), formulate the refined local sampling meshes $\mathcal{T}_{h^{\prime}}$, $\left\{r_{j}\right\}_{j \in \mathscr{T}_{2}^{\prime}}$ and $\left\{U_{j}\right\}_{j \in \mathscr{T}_{1}^{\prime}}, j=1,2, \ldots, l$.

b) For a local tuneup given in (4.10)-(4.11), calculate the far-field pattern $\widehat{a}_{k}(\hat{x}, d)$ according to $(4.12)$.

c) Using $\widehat{a}_{k}(\hat{x}, d)$ obtained in b) as the far-field data, apply Scheme I to locate the significant local maximum points on $\mathcal{T}_{h} \backslash \cup_{j=1}^{l} \mathcal{N}_{1}^{j}$.

d) Repeat b) and c) for all possible local tuneups relative to $\left\{\mathcal{T}_{h^{\prime}},\left\{r_{j}\right\}_{j \in \mathscr{T}_{2}^{\prime}},\left\{U_{j}\right\}_{j \in \mathscr{T}_{1}^{\prime}}\right\}$. The clustered local maximum points on $\mathcal{T}_{h} \backslash \cup_{j=1}^{l} \mathcal{N}_{1}^{j}$ are the positions corresponding to the scatterer components of $\Omega^{s, t}$.

e) Update $\widehat{\Omega}^{r}$ to the local tuneup $\widehat{\widehat{\Omega^{r}}}$ which generates the clustered local maximum points in d). 


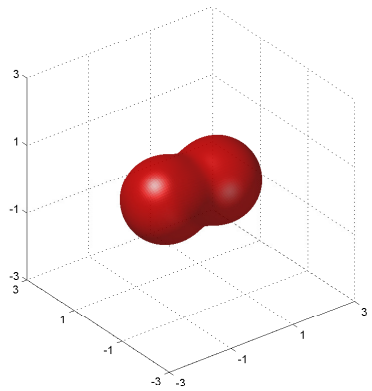

$0^{o}$

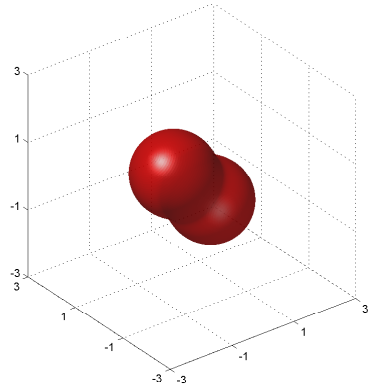

$45^{\circ}$

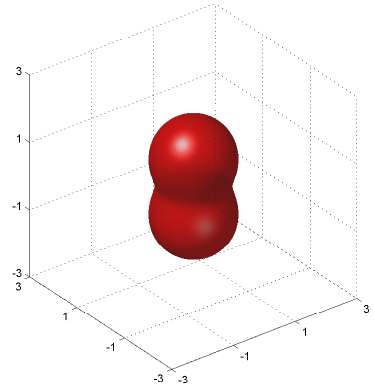

$90^{\circ}$

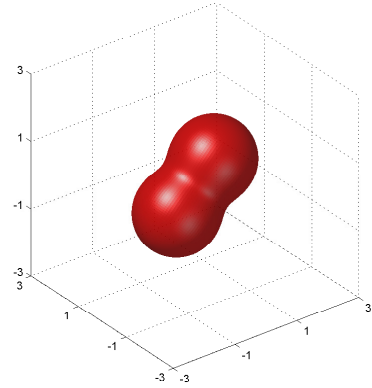

$135^{\circ}$

Figure 14: Basic Scatterers: Peanut (multiscale)

Remark 4.1. By concatenating Schemes I and II with the local tuning technique, Scheme III can effectively locate multiple multiscale scatterers. For the practical consideration, one can easily see from our discussions that as long as the scattering strengths from the small scatterer components of $\Omega^{s, t}$ are more significant than the measurement noise involved, Scheme III can produce reasonable reconstructions for multiple multiscale scatterers.

\subsection{Numerical experiments}

In this subsection, we test some 3D multiscale imaging problem using Scheme III. The wave number $k$ is chosen to be 5 and the incident direction is $d=(0,0,-1)$. The synthetic data are obtained for the revolving solids of the $2 \mathrm{D}$ shapes $\mathbf{K}$ and $\mathbf{P}$ along the $x$-axis, which are for short still denoted by $\mathbf{K}$ and $\mathbf{P}$ without ambiguities. As for each reference component, we rotate it every 90 degrees in the $x-y, y-z$ and $z$-x planes; see, e.g., four different orientations of the peanut in the $z-x$ plane are shown in Fig. 14. Three different sizes of the reference components are tested, namely scaled by a factor of $0.3,1$ and 1.5 respectively.

Example 6. We consider a 3D multiscale scatterer consisting of two components, a small sound-hard kite scaled by a factor 0.3 located at $(-5,0,5)$ and a large sound-hard peanut with no scaling and located at $(5,0,-5)$, see Fig. 15.

In the first stage, we extract the information of the regular-size component using the indicator

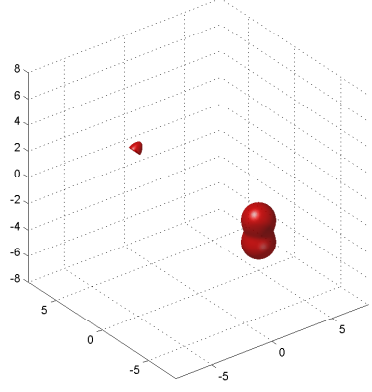

True scatterer.

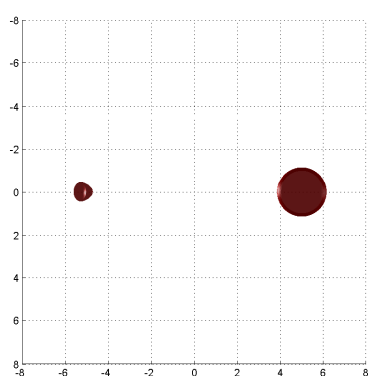

$x-y$ plane

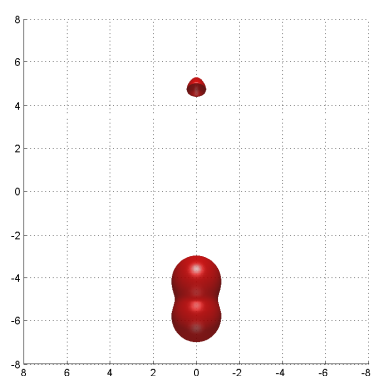

$y$-z plane

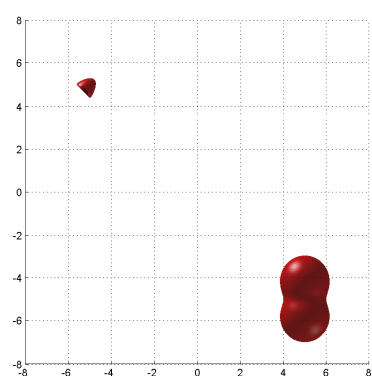

$x-z$ plane

Figure 15: True scatterer components in Example 6. 


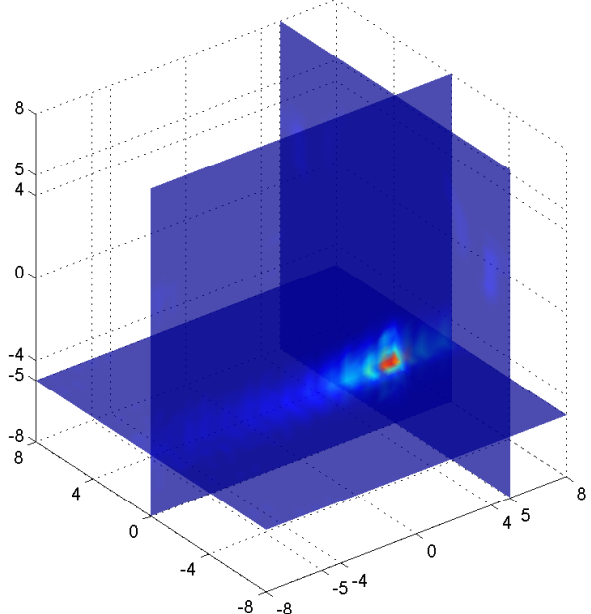

(a)

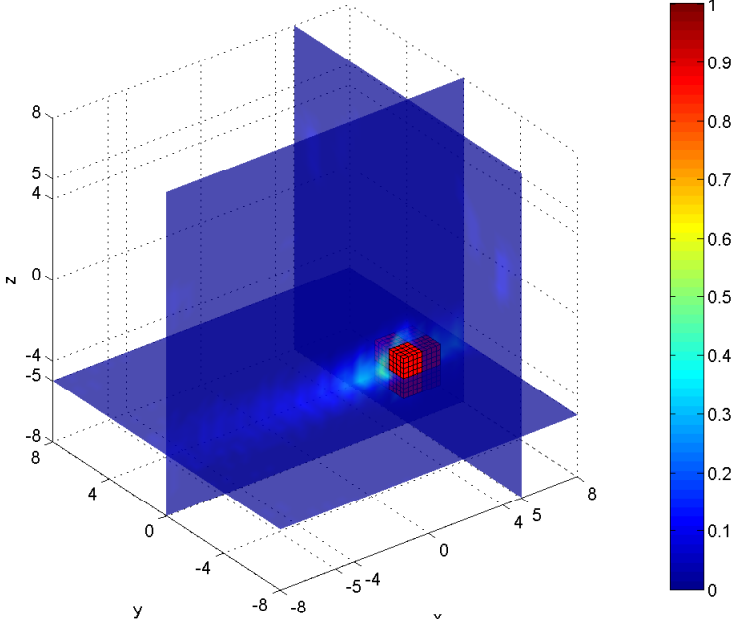

(b)

Figure 16: (a) Imaging of the 3D multi-scale scatterer in the first stage. (b) A local resampling mesh.

function of Scheme II by computing the inner product with a priori known far-field patterns associated with those reference scatterer components with different orientations and sizes. We can find the approximate position of the larger peanut component of regular size when the reference scatterer is at its upright position, see Fig. 16(a).

Next, we adopt the local tuning (resampling) technique discussed in Section 4.1 to search a small cubic mesh around the rough position of the peanut determined by the local maximum, which is shown in Fig. 16(b).

In the final stage, the location of the smaller component can be obtained by performing a local searching of each grid point in the cubic mesh. In Fig. 17, as the search grid points approach gradually from $(4.8,0,-5)$ to $(5,0,-5)$ (from left to right), the value distribution of the indicator function in Scheme I displays an interesting change of the highlighted position. In the right plot of Fig. 17, the red dot indicates an approximate position of the smaller kite component, which agrees with the exact one very well. In such a way, the smaller sound-hard kite could be positioned, and it helps us finely tune the position of the peanut and update it to be around $(5,0,-5)$. They could now be well approximated in Fig. 18 by combining the relevant shape, scale and size information hidden in the reference data.

\section{Acknowledgements}

The authors would like to thank the anonymous referees and the board member for many insightful and constructive comments and suggestions, which have significantly improved the quality of the paper. The work of Jingzhi Li was supported by the NSF of China (No. 11201453 and 91130022). The work of Hongyu Liu was supported by NSF grant (DMS 1207784). The work of Jun Zou was supported by Hong Kong RGC grant (Project 404611). 

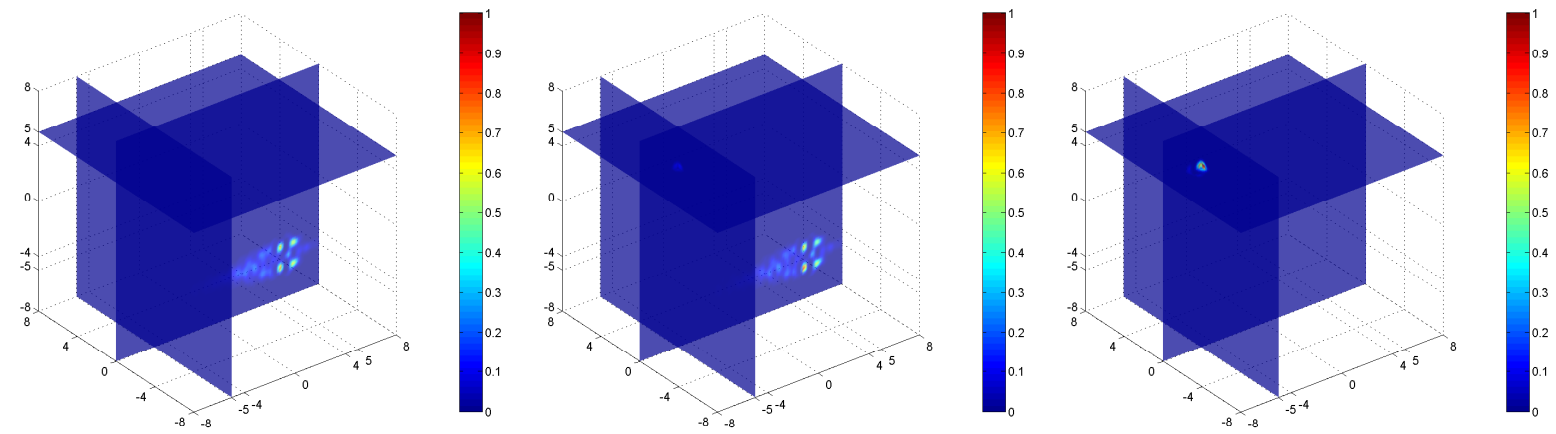

Figure 17: Example 6. Indicating behavior of local re-sampling technique. From left to right: Imaging of indicator function value distribution at the sampling points $(4.8,0,-5),(4.9,0,-5)$ and $(5,0,-5)$, respectively.
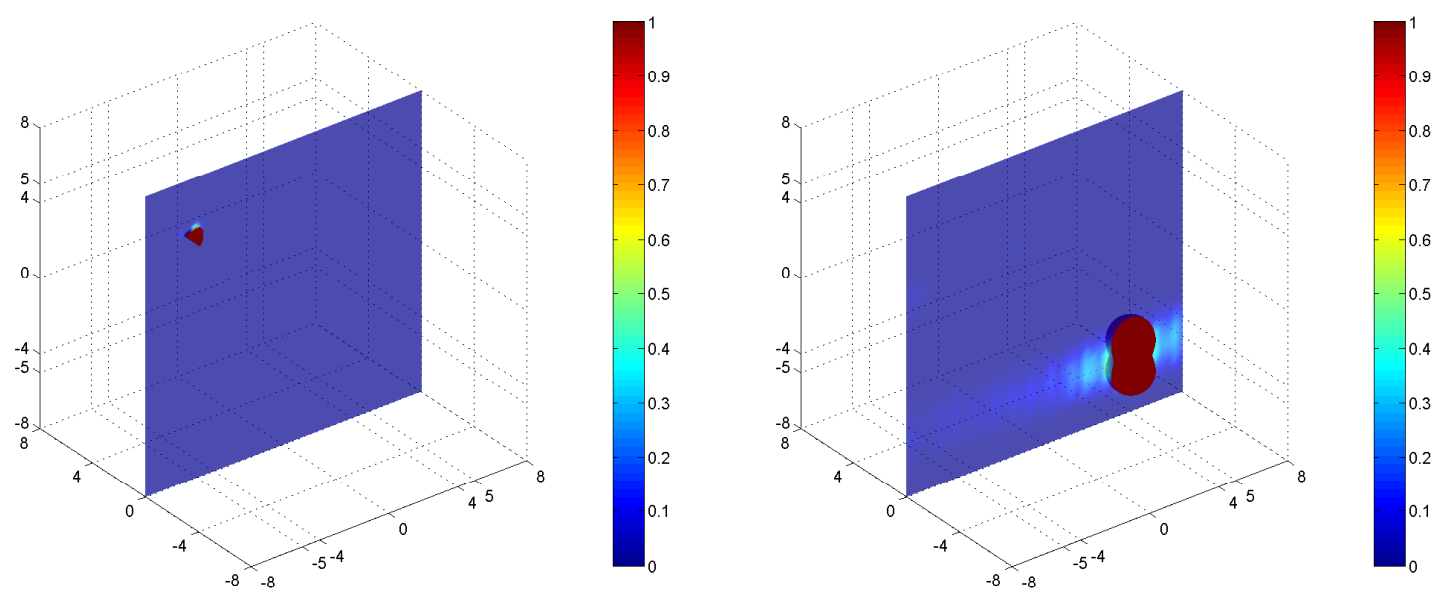

Figure 18: Example 6. From left to right: local sampling procedure at the sampling points $(-4.8,0,5),(-4.9,0,5)$ and $(5,-5)$, respectively. Imaging of the position of the reconstructed kite component. 


\section{References}

[1] H. Ammari, T. Boulier, J. Garnier, W. Jing, H. Kang, and H. Wang, Target identification using dictionary matching of generalized polarization tensors, Found. Comput. Math., DOI 10.1007/s10208-013-9168-6.

[2] H. Ammari, T. Boulier and J. Garnier, Modeling active electrolocation in weakly electric fish, SIAM J. Imaging Sci., 5 (2013), 285-321.

[3] H. Ammari, J. Chen, Z. Chen, D. Volkov, and H. Wang, Detection and classification from electromagnetic induction data, arXiv:1308.6027.

[4] R. Aramini, M. Brignone, and M. Piana, The linear sampling method without sampling, Inverse Problems, 22 (2006), 2237-2254.

[5] H. Ammari, P. Garapon, F. Jouve, H. Kang, M. Lim, and S. Yu, A new optimal control approach for the reconstruction of extended inclusions, SIAM J. Control Optim., 51 (2013), 1372-1394.

[6] H. Ammari, J. Garnier, V. Jugnon, and H. Kang, Stability and resolution analysis for a topological derivative based imaging functional, SIAM J. Control Optim. 50 (2012), no. 1, 48-76.

[7] H. Ammari, J. Garnier, H. Kang, M. Lim, and K. Solna, Multistatic imaging of extended targets, SIAM J. Imaging Sci. 5 (2012), no. 2, 564-600.

[8] H. Ammari, J. Garnier, H. Kang, W.K. Park, and K. Solna, Imaging schemes for perfectly conducting cracks, SIAM J. Appl. Math., 71 (2011), no. 1, 68-91.

[9] H. Ammari, J. Garnier, and K. Solna, A statistical approach to target detection and localization in the presence of noise, Waves Random Complex Media, 22 (2012), no. 1, 40-65.

[10] H. Ammari, E. Iakovleva and D. Lesselier, A MUSIC algorithm for locating small inclusions buried in a half-space from the scattering amplitude at a fixed freuqency, Multiscale Model. Simul., 3 (2005), 597-628.

[11] H. Ammari, E. Iakovleva, D. Lesselier and G. Perrusson, MUSIC-type electromagnetic imaging of a collection of small three-dimensional inclusions, SIAM J. Sci. Comput., 29 (2007), $674-709$.

[12] H. Ammari and H. Kang, Reconstruction of small inhomogeneities from boundary measurements, Lecture Notes in Mathematics, 1846, Springer-Verlag, Berlin, 2004.

[13] H. Ammari and H. Kang, Polarization and moment tensors. With applications to inverse problems and effective medium theory, Applied Mathematical Sciences, Springer, New York, 2007.

[14] H. Ammari, M. P. Tran and H. Wang, Shape identification and classification in echolocation, arXiv:1308.5625.

[15] C. Bellis, M. Bonnet and F. Cakoni, Acoustic inverse scattering using topological derivative of far-field measurements-based $L^{2}$ cost functionals, Inverse Problems, 29 (2013), 075012. 
[16] M. Bonnet, Inverse acoustic scattering by small-obstacle expansion of a misfit function, Inverse Problems, 24 (2008), 035022.

[17] M. Bonnet and B. B. Guzina, Sounding of finite solid bodies by way of topological derivative, Int. J. Numer. Methods Eng., 61 (2004), 2344-2373.

[18] F. Cakoni and D. Colton, Qualitative Methods in Inverse Scattering Theory: An Introduction, Springer, 2005.

[19] F. Cakoni, D. Colton, and P. Monk, The Linear Sampling Method in Inverse Electromagnetic Scattering, Philadelphia: SIAM, 2011.

[20] D. Colton, J. Coyle and P. Monk, Recent developments in inverse acoustic scattering theory, SIAM Rev., 42 (2000), 369-414.

[21] D. Colton and A. Kirsch, A simple method for solving inverse scattering problems in the resonance region, Inverse Problems, 12 (1996), 383-393.

[22] D. Colton and R. Kress, Inverse Acoustic and Electromagnetic Scattering Theory, 2nd Ed., Springer, New York, 1998.

[23] G. Dassios and R. Kleinman, Low Frequency Scattering, Clarendon Press, Oxford, 2000.

[24] R. Griesmaier, Multi-frequency orthogonality sampling for inverse obstacle scattering problems, Inverse Problems, 27 (2011), 085005.

[25] B. B. Guzina and M. Bonnet, Small-inclusion asymptotic of misfit functionals for inverse problems in acoustics, Inverse Problems, 22 (2006), 1761-1785.

[26] K. Ito, B. Jin and J. Zou, A direct sampling method to an inverse medium scattering problem, Inverse Problems, 28 (2012), 025003.

[27] V. Isakov, Inverse Problems for Partial Differential Equations, 2nd edition, Applied Mathematical Sciences, 127, Springer-Verlag, New York, 2006.

[28] A. Kirsch and N. Grinberg, The Factorization Method for Inverse Problems, Oxford University Press, Oxford, 2008.

[29] I. Kocyigit, H. Y. Liu and H. Sun, Regular scattering patterns from near-cloaking devices and their implications for invisibility cloaking, Inverse Problems, 29 (2013), 045005.

[30] V. I. Lebedev and D. N. Laikov, A quadrature formula for the sphere of the 131st algebraic order of accuracy, Doklady Mathematics, 59 (1999), pp. 477-481.

[31] J. Li, H. Y. Liu, Z. Shang and H. Sun, Two single-shot methods for locating multiple electromagnetic scatterers, SIAM J. Appl. Math., 73 (2013), 1721-1746.

[32] J. Li, H. Y. Liu and Q. Wang, Locating multiple multiscale electromagnetic scatterers by a single far-field measurement, SIAM J. Imaging Sci., 6 (2013), 2285-2309.

[33] J. Li, H. Liu, and J. Zou, Strengthened linear sampling method with a reference ball, SIAM J. Sci. Comp., 31 (2009), 4013-4040. 
[34] H. Y. Liu, Virtual reshaping and invisibility in obstacle scattering, Inverse Problems, 25 (2009), 045006.

[35] H. Y. Liu, On recovering polyhedral scatterers with acoustic far-field measurements, IMA J. Appl. Math, 74 (2009), 264-272.

[36] H. Y. Liu, Z. Shang, H. Sun and J. Zou, Singular perturbation of reduced wave equation and scattering from an embedded obstacle, J. Dynam. Diff. Eqn., 24 (2012), 803-821.

[37] H. Y. Liu and J. Zou, Uniqueness in an inverse acoustic obstacle scattering problem for both sound-hard and sound-soft polyhedral scatterers, Inverse Problems, 22 (2006), 515-524.

[38] H. Y. Liu and J. Zou, On uniqueness in inverse acoustic and electromagnetic obstacle scattering problems, J. Phys.: Conf. Series, 124 (2008), 012006.

[39] P. A. Martin, Multiple Scattering: Interaction of Time-Harmonic Waves with N Obstacles, Cambridge Univ. Press, Cambridge, 2006.

[40] W. McLean, Strongly Elliptic Systems and Boundary Integral Equations, Cambridge University Press, Cambridge, 2000.

[41] J.-C. Nédélec, Acoustic and Electromagnetic Equations: Integral Representations for Harmonic Problems, Springer-Verlag, New York, 2001.

[42] R. Pike and P. Sabatier eds., Scattering : Scattering and Inverse Scattering in Pure and Applied Science, Academic Press, 2002.

[43] R. Potthast, A study on orthogonality sampling, Inverse Problems, 26 (2010), 074015.

[44] R. Potthast, A survey on sampling and probe methods for inverse problems, Inverse Problems, 22 (2006), R1-R47.

[45] R. Potthast, Point Sources and Multipoles in Inverse Scattering Theory, CRC Press, 2001.

[46] G. Uhlmann, ed., Inside Out: Inverse Problems and Applications, MSRI Publications, Vol. 47, Cambridge University Press, 2003. 